\title{
DIGITALCOMMONS
}

— @WAYNESTATE —

Wayne State University

6-1-2009

\section{Variational Analysis in Semi-Infinite and Infinite Programming, I: Stability of Linear Inequality Systems of Feasible Solutions}

\section{J. Cánovas}

Miguel Hernández University of Elche, Alicante, Spain, canovas@umh.es

M A. Lopez

University of Alicante, Spain, marco.antonio@ua.es

Boris S. Mordukhovich

Wayne State University, boris@math.wayne.edu

J Parra

Miguel Hernández University of Elche, Alicante, Spain, parra@umh.es

\section{Recommended Citation}

Cánovas, M J.; Lopez, M A.; Mordukhovich, Boris S.; and Parra, J, "Variational Analysis in Semi-Infinite and Infinite Programming, I: Stability of Linear Inequality Systems of Feasible Solutions" (2009). Mathematics Research Reports. Paper 67.

http://digitalcommons.wayne.edu/math_reports/67 


\title{
VARIATIONAL ANALYSIS IN SEMI-INFINITE AND INFINITE PROGRAMMING, I: STABILITY OF LINEAR INEQUALITY SYSTEMS OF FEASIBLE SOLUTIONS
}

\author{
M. J. CÁNOVAS, M. A. LÓPEZ, B. S. MORDUKHOVICH \\ and J. PARRA
}

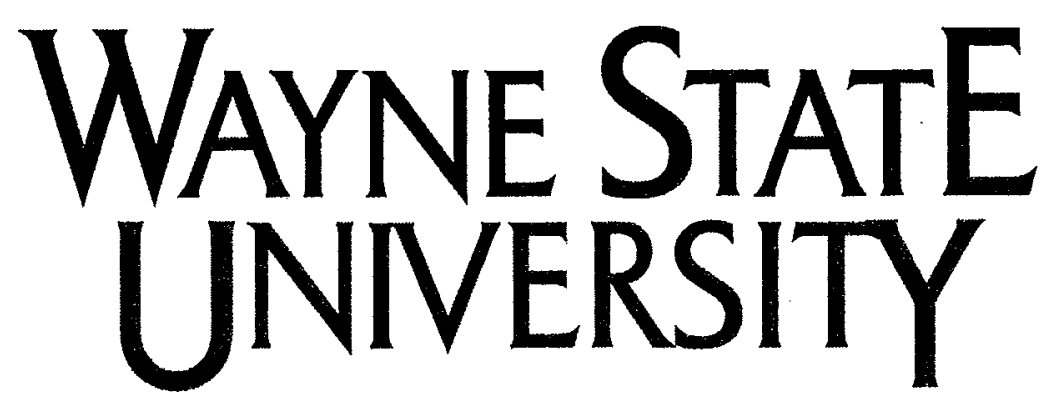

Detroit, MI 48202

Department of Mathematics

Research Report

\section{Series}

\#6

This research was partly supported by the USA National Science Foundation 


\title{
VARIATIONAL ANALYSIS IN SEMI-INFINITE AND INFINITE PROGRAMMING, I: STABILITY OF LINEAR INEQUALITY SYSTEMS OF FEASIBLE SOLUTIONS ${ }^{1}$
}

\author{
M. J. CÁNOVAS ${ }^{2}$, M. A. LÓPEZ ${ }^{3}$, B. S. MORDUKHOVICH ${ }^{4}$ and J. PARRA ${ }^{2}$
}

\begin{abstract}
This paper concerns applications of advanced techniques of variational analysis and generalized differentiation to parametric problems of semi-infinite and infinite programming, where decision variables run over finite-dimensional and infinite-dimensional spaces, respectively. Part I is primarily devoted to the study of robust Lipschitzian stability of feasible solutions maps for such problems described by parameterized systems of infinitely many linear inequalities in Banach spaces of decision variables indexed by an arbitrary set $T$. The parameter space of admissible perturbations under consideration is formed by all bounded functions on $T$ equipped with the standard supremum norm. Unless the index set $T$ is finite, this space is intrinsically infinite-dimensional (nonreflexive and nonseparable) of the $l_{\infty}$-type. By using advanced tools of variational analysis and exploiting specific features of linear infinite systems, we establish complete characterizations of robust Lipschitzian stability entirely via their initial data with computing the exact bound of Lipschitzian moduli. - A crucial part of our analysis addresses the precise computation of the coderivative of the feasible set mapping and its norm. The results obtained are new in both semi-infinite and infinite frameworks.
\end{abstract}

Key words. semi-infinite and infinite programming, variational analysis, linear infinite inequality systems, robust stability, generalized differentiation, coderivatives

AMS subject classification. 90C34, 90C05, 49J52, 49J53, 65F22

\section{Introduction}

This paper mainly deals with parameterized infinite systems of linear inequalities

$$
\mathcal{F}(p):=\left\{x \in X \mid\left\langle a_{t}^{*}, x\right\rangle \leq b_{t}+p_{t}, t \in T\right\}, \quad p=\left(p_{t}\right)_{t \in T}
$$

with an arbitrary index set $T$, where $x \in X$ is a decision variable belonging to an arbitrary Banach space $X$ (which may be finite-dimensional), and where $p=\left(p_{t}\right)_{t \in T} \in P$ is a functional parameter taking values in the prescribed Banach space $P$ of perturbations specified below. Infinite inequality systems of this type are important in various areas of mathematics and applications, while our primary interest to them is driven by applications to problems of semi-infinite and infinite programming corresponding to finite-dimensional and infinite-dimensional decision spaces $X$, respectively; see, e.g., books $[1,5,19,37]$ and

\footnotetext{
${ }^{1}$ This research was partially supported by grants MTM2005-08572-C03 (01-02) from MEC (Spain) and FEDER (EU), and MTM2008-06695-C03 (01-02) from MICINN (Spain).

${ }^{2}$ Center of Operations Research, Miguel Hernández University of Elche, 03202 Elche (Alicante), Spain (canovas@umh.es, parra@umh.es).

${ }^{3}$ Department of Statistics and Operations Research, University of Alicante, 03080 Alicante, Spain(marco.antonio@ua.es).

${ }^{4}$ Department of Mathematics, Wayne State University, Detroit, MI 48202, USA (boris@math.wayne.edu). The research of this author was partially supported by the National Science Foundation (USA) under grant DMS-0603846.
} 
the references therein. Some applications of the results obtained in this paper to necessary optimality conditions for semi-infinite and infinite programs could be found in [11].

The data of (1.1) are given as follows:

- $a_{t}^{*} \in X^{*}$ for all $t \in T$, where the space $X^{*}$ is topologically dual to $X$ with the canonical pairing $\langle\cdot, \cdot\rangle$ between $X$ and $X^{*}$. If no confusion arises, we use the same notation $\|\cdot\|$ for the given norm in $X$ and the corresponding dual norm in $X^{*}$ defined by

$$
\left\|x^{*}\right\|:=\sup \left\{\left\langle x^{*}, x\right\rangle \mid\|x\| \leq 1\right\}, \quad x^{*} \in X^{*} .
$$

We always assume that $a_{t}^{*}$ are fixed and arbitrary in $X^{*}$ for all $t \in T$.

- $b_{t} \in \mathbb{R}$ for all $t \in T$. We identify the collection $\left\{b_{t} \mid t \in T\right\}$ with the real-valued function $b: T \rightarrow \mathbb{R}$ and assume that it is fixed and arbitrary.

- $p_{t}=p(t) \in \mathbb{R}$ for all $t \in T$. These functional parameters $p: T \rightarrow \mathbb{R}$ are our varying perturbations, which are taken from the Banach parameter space $P:=l_{\infty}(T)$ of all bounded functions on $T$ with the supremum norm

$$
\|p\|_{\infty}:=\sup _{t \in T}\left|p_{t}\right|=\sup \{|p(t)| \mid t \in T\}
$$

(see, e.g., [17]), where the subscript " $\infty$ " is omitted if no confusion arises. When the index set $T$ is compact (which is not assumed in this paper) and the perturbations $p(\cdot)$ are restricted to be continuous on $T$, the maximum is realized in (1.2), and thus the parameter space $l_{\infty}(T)$ reduces to the classical space $\mathcal{C}(T)$ of continuous functions over a compact set.

The primary goal of this paper is to obtain comprehensive characterizations of robust Lipschitzian stability of infinite inequality systems (1.1) expressed entirely in terms of their initial data. By robust Lipschitzian stability we understand the fulfillment of the so-called Lipschitz-like (known also as Aubin) property of the mapping $\mathcal{F}(p)$ in (1.1) around the reference point. This property is stable with respect to small perturbations of parameters and is crucial for both qualitative and quantitative/numerical aspects of optimization theory and applications; see, e.g., [31, 32, 35] and Section 2 for more details and references.

To establish constructive characterizations of robust Lipschitzian stability in this paper and to derive efficient optimality conditions for semi-infinite and infinite programs in [11], we develop an advanced approach of variational analysis based on generalized differentiation. To the best of our knowledge, this approach is new in the literature on semi-infinite and infinite programming despite many publications related to various stability properties and applications of linear infinite inequality systems, most of which concern the case of finite-dimensional spaces $X$ of decisions variables (i.e., in the semi-infinite programming framework); see, e.g., $[1,19]$ for comprehensive overviews on this field and also [5] confined to the parameter space of continuous perturbations $P=\mathcal{C}(T)$ when the index set $T$ is a compact Hausdorff space. We refer the reader to [12] for the study of qualitative stability (formalized through certain semicontinuity properties of feasible solution and optimal solution mappings) in the framework of $X=\mathbb{R}^{n}$, an arbitrary index set $T$, and arbitrary perturbations. In the same semi-infinite context, for a quantitative perspective (through 
Lipschitzian properties), the reader is addressed to [7], and to [6] for the case of continuous perturbations. Let us mention the recent paper [14] addressing the case of infinite linear programming from the viewpoint of qualitative stability. We also refer the reader to, e.g., $[8,9,13,15,18,24,25]$ for the study of convex semi-infinite and infinite programs and to $[21,22,37]$ for their smooth nonlinear counterparts.

The approach of this paper is mainly based on coderivative analysis of the parametric linear infinite inequality systems $\mathcal{F}$ in $(1.1)$, which eventually leads us to complete characterizations of robust Lipschitzian stability for the parametric sets of feasible solutions in infinite/semi-infinite programming expressed entirely via their initial data with precise computing the exact bound of Lipschitzian moduli.

Coderivatives of set-valued mappings introduced in [27] have been well recognized as a powerful tool of variational analysis and its numerous applications, particularly to problems of optimization and control; see, e.g., books $[3,23,31,32,33,35,36]$ and the references therein. However, we are not familiar with any implementation of coderivatives in problems of infinite or semi-infinite programming as well as with their application to analyze stability of linear infinite inequality systems of type (1.1) in finite or infinite dimensions.

The power of coderivatives in variational analysis and its applications comes, first of all, from the possibility to obtain in their terms verifiable pointwise characterizations of robust Lipschitzian properties of set-valued mappings (as well as of the equivalent properties of metric regularity and linear openness for the inverse mappings) and to derive necessary optimality conditions in rather general settings. These developments are strongly supported by comprehensive pointwise coderivative calculus based on variational/extremal principles of advanced variational analysis; see [31, 32] and the references therein. However, a number of results in this vein are limited in infinite dimensions. In particular, the available coderivative characterizations of the Lipschitz-like property of closed-graph mappings $F: Z \rightrightarrows Y$ obtained in [31, Theorem 4.10] require that both spaces $Z$ and $Y$ are Asplund (i.e., every separable subspace of them has a separable dual) while the precise coderivative formula for computing the exact Lipschitzian bound is established therein via the coderivative norm under the finite-dimensionality assumption on $Z$. But this is never the case for our infinite inequality system $\mathcal{F}: P \rightrightarrows X$ from $(1.1)$, where the parameter space $(Z=) P=l_{\infty}(T)$ is always infinite-dimensional and not Asplund unless the index set $T$ is finite!

This paper contains new and fairly comprehensive results in the aforementioned directions for the infinite/semi-infinite systems under consideration, which essentially take into account underlying specific features of the infinite inequality constraints (1.1) largely related to the possibility of employing an appropriate extended version of the fundamental Farkas Lemma for infinite systems of linear inequalities in general Banach spaces.

The rest of paper is organized as follows. Section 2 presents some preliminary material from convex and variational analysis widely used in formulations and proofs of the subsequent main results. In Section 3 we provide precise calculations of the basic coderivative $D^{*} \mathcal{F}$ and its norm at the reference/nominal point for the feasible solution map $\mathcal{F}: l_{\infty}(T) \rightrightarrows X$ in (1.1) via the initial data of $\mathcal{F}$ in the general case of an arbitrary index set $T$ and an arbitrary Banach space $X$ of decision variables.

Section 4 is devoted to deriving coderivative characterizations of robust stability for the 
feasible solution system (1.1) of infinite inequalities with an arbitrary index set $T$, which are explicitly expressed in terms of the initial data $\left\{a_{t}^{*}, b_{t}, t \in T\right\}$. We establish verifiable criteria (i.e., necessary and sufficient conditions) for the fulfillment of the Lipschitz-like (and hence the classical local Lipschitzian) property of $\mathcal{F}$ around the reference points and derive furthermore the precise formulas for computing the exact bounds of Lipschitzian moduli in the case of general Banach spaces $X$. It is worth mentioning that the criteria and exact bound formulas obtained in this section are represented in the conventional coderivative form of variational analysis as in [31, Theorem 4.10] for the case of abstract set-valued mappings, but with no Asplund space and finite-dimensionality requirements imposed therein. In fact, the latter requirements are never satisfied for the infinite linear inequality systems (1.1) under consideration in either infinite programming or semi-infinite programming framework.

Our notation is basically standard and conventional in the areas of variational analysis and infinite/semi-infinite programming; see, e.g., [19, 31, 35]. Unless otherwise stated, all the spaces under consideration are Banach with the corresponding norm $\|\cdot\|$. Recall that $w^{*}$ indicates the weak* topology of a dual space, and we use the symbol $w^{*}$-lim for the weak* topological limit, which generally means the weak* convergence of nets denoted usually by $\left\{x_{\nu}^{*}\right\}_{\nu \in \mathcal{N}}$. In the case of sequences we use the standard notation $\mathbb{N}:=\{1,2, \ldots\}$ for the collection of all natural numbers.

Given a subset $\Omega \subset Z$ of a Banach space, the symbols int $\Omega, \operatorname{cl} \Omega, \operatorname{co} \Omega$, and cone $\Omega$ stand, respectively, for the interior, closure, convex hull, and conic convex hull of $\Omega$; the notation $\mathrm{cl}^{*} \Theta$ signifies the weak ${ }^{*}$ closure of a subset $\Theta \subset Z^{*}$ in the dual space. Given a set-valued mapping $F: Z \Rightarrow Y$, we denote its domain, graph, and inverse by, respectively,

$$
\operatorname{dom} F=\{z \in Z \mid F(z) \neq \emptyset\}, \quad \operatorname{gph} F:=\{(z, y) \in Z \times Y \mid y \in F(z)\},
$$

and $F^{-1}(y):=\{z \in Z \mid(z, y) \in \operatorname{gph} F\}$. Considering finally an arbitrary index set $T$, let $\mathbb{R}^{T}$ be the product space of $\lambda=\left(\lambda_{t} \mid t \in T\right)$ with $\lambda_{t} \in \mathbb{R}$ for all $t \in T$, let $\mathbb{R}^{(T)}$ be the collection of $\lambda \in \mathbb{R}^{T}$ such that $\lambda_{t} \neq 0$ for finitely many $t \in T$, and let $\mathbb{R}_{+}^{(T)}$ be the positive cone in $\mathbb{R}^{(T)}$ defined by

$$
\mathbb{R}_{+}^{(T)}:=\left\{\lambda \in \mathbb{R}^{(T)} \mid \lambda_{t} \geq 0 \text { for all } t \in T\right\} .
$$

\section{Basic Definitions and Preliminaries}

In this section we discuss the basic notions and tools needed for our subsequent analysis and results. As mentioned in Section 1, a major attention of this paper is paid to robust Lipschitzian stability of the feasible solution map given by (1.1). By such a robust stability we understand Lipschitzian behavior around (i.e., in a neighborhood) of the reference point. The most natural formalization of this behavior widely recognized in variational (as well as in general nonlinear) analysis is known as the Lipschitz-like or Aubin property, which can be viewed as a graphical localization (in the set-valued case) of the classical local Lipschitzian property of single-valued and set-valued mappings. 
Given a set-valued mapping $F: Z \rightrightarrows Y$ between Banach spaces, we say the $F$ is Lipschitz-like around $(\bar{z}, \bar{y}) \in \operatorname{gph} F$ with modulus $\ell \geq 0$ if there are neighborhoods $U$ of $\bar{z}$ and $V$ of $\bar{y}$ such that

$$
F(z) \cap V \subset F(u)+\ell\|z-u\| \mathbb{B} \text { for any } z, u \in U
$$

where $\mathbb{B}$ stands for the closed unit ball in the space in question. The infimum of moduli $\{\ell\}$ over all the combinations of $\{\ell, U, V\}$ satisfying (2.1) is called the exact Lipschitzian bound of $F$ around $(\bar{z}, \bar{y})$ and is labeled as $\operatorname{lip} F(\bar{z}, \bar{y})$. If $V=Y$ in (2.1), this relationship signifies the classical (Hausdorff) local Lipschitzian property of $F$ around $\bar{z}$ with the exact Lipschitzian bound denoted by $\operatorname{lip} F(\bar{z})$ in this case.

It is worth mentioning that the Lipschitz-like property of an arbitrary mapping $F: Z \rightrightarrows Y$ between Banach spaces is equivalent to other two fundamental properties in nonlinear analysis but for the inverse mapping $F^{-1}: Y \rightrightarrows Z$; namely, to the metric regularity of $F^{-1}$ and to the linear openness of $F^{-1}$ around $(\bar{y}, \bar{z})$, with the corresponding relationships between their exact bounds (see, e.g., $[20,31,35]$ ).

It is well known that the exact Lipschitzian bound of $F$ around $(\bar{z}, \bar{y})$ admits the following limiting representation via the distance function to a set;

$$
\operatorname{lip} F(\vec{z}, \bar{y})=\limsup _{(z, y) \rightarrow(\bar{z}, \bar{y})} \frac{\operatorname{dist}(y ; F(z))}{\operatorname{dist}\left(z ; F^{-1}(y)\right)}
$$

where $\inf \emptyset=\infty$ (and hence $\operatorname{dist}(x ; \emptyset)=\infty$ ) as usual and where $0 / 0:=0$. We have accordingly that lip $F(\bar{z}, \bar{y})=\infty$ if $F$ is not Lipschitz-like around $(\bar{z}, \bar{y})$.

A remarkable fact consists of the possibility to characterize pointwisely the (derivativefree) Lipschitz-like property of $F$ around $(\bar{z}, \bar{y})$-and hence its local Lipschitzian, metric regularity, and linear openness counterparts-in terms of a dual-space construction of generalized differentiation called the coderivative of $F$ at $(\bar{z}, \bar{y}) \in \operatorname{gph} F$. The latter is a positively homogeneous multifunction $D^{*} F(\bar{z}, \bar{y}): Y^{*} \rightrightarrows Z^{*}$ defined by

$$
D^{*} F(\bar{z}, \bar{y})\left(y^{*}\right):=\left\{z^{*} \in Z^{*} \mid\left(z^{*},-y^{*}\right) \in N((\bar{z}, \bar{y}) ; \operatorname{gph} F)\right\}, \quad y^{*} \in Y^{*},
$$

where $N(\cdot ; \Omega)$ stands for the collection of generalized normals to a set at a given point known as the basic, or limiting, or Mordukhovich normal cone; see, e.g., [26, 31, 35, 36] and the references therein. When both $Z$ and $Y$ are finite-dimensional, it is proved in [28] (cf. also [35, Theorem 9.40]) that a closed-graph mapping $F: Z \rightrightarrows Y$ is Lipschitz-like around $(\bar{z}, \bar{y}) \in \operatorname{gph} F$ if and only if

$$
D^{*} F(\bar{z}, \bar{y})(0)=\{0\}
$$

and that the exact Lipschitzian bound of moduli $\{\ell\}$ in (2.1) is computed by

$$
\operatorname{lip} F(\bar{z}, \bar{y})=\left\|D^{*} F(\bar{z}, \bar{y})\right\|:=\sup \left\{\left\|z^{*}\right\| \mid z^{*} \in D^{*} F(\bar{z}, \bar{y})\left(y^{*}\right),\left\|y^{*}\right\| \leq 1\right\} .
$$

The situation is significantly more involved in infinite dimension. It is proved in [29] (see also [31, Theorem 4.10]) that a closed-graph mapping $F: Z \rightrightarrows Y$ is Lipschitz-like around $(\bar{z}, \bar{y}) \in \operatorname{gph} F$ if and only if the coderivative condition (2.4) holds in terms of the so-called 
"mixed coderivative" (which reduces to (2.3) in finite dimensions and the setting considered in this paper) together with a certain "partial sequential normal compactness" condition (which automatically holds in finite dimensions and in the setting of this paper) provided that both spaces $Z$ and $Y$ are Asplund. The latter property is defined in Section 1; we also refer the reader to $[17,31,34]$ for more details and various characterizations of this remarkable and well investigated subclass of Banach spaces that includes, in particular, all reflexive ones while does not include, e.g., the classical spaces $\mathcal{C}, l_{1}, L_{1}, l_{\infty}$, and $L_{\infty}$.

The situation is even more complicated with infinite-dimensional extensions of the exact bound formula in (2.5). The aforementioned results of $[29,31]$ give merely upper and lower estimates for $\operatorname{lip} F(\bar{z}, \bar{y})$, which ensure the precise equality in (2.5) in our setting here provided that $Y$ is Asplund while $Z$ is finite-dimensional.

The set-valued mapping $F: Z \rightrightarrows Y$ considered in this paper is $\mathcal{F}: l_{\infty}(T) \rightrightarrows X$ defined by the infinite system of linear inequalities (1.1); in what follows we always assume that the index set $T$ is infinite, which is a characteristic feature of infinite and semi-infinite programs. In this setting the domain/parameter space $Z=l_{\infty}(T)$ must be infinite-dimensional Banach that is never Asplund. Also, we do not suppose in this paper that our decision space $X$ is anything but arbitrary Banach.

In this general setting for (1.1) we show that the coderivative condition (2.4) is necessary and sufficient for the Lipschitz-like property of $F=\mathcal{F}$ around the reference/nominal solution $(\vec{p}, \vec{x}) \in \operatorname{gph} \mathcal{F}$ and (2.5) is a precise formula for computing the exact Lipschitzian bound lip $\mathcal{F}(\bar{p}, \bar{x})$. This is exactly what we have in finite dimensions, while it is far removed from being a part of the infinite-dimensional variational theory in [31]. Moreover, we express the relationships in (2.4) and (2.5) explicitly in terms of the initial data of (1.1).

To proceed further, observe that the graph

$$
\operatorname{gph} \mathcal{F}=\left\{(p, x) \in l_{\infty}(T) \times X \mid\left\langle a_{t}^{*}, x\right\rangle \leq b_{t}+p_{t} \text { for all } t \in T\right\}
$$

of the mapping $\mathcal{F}: l_{\infty}(T) \rightrightarrows X$ in (1.1) is convex. Hence the basic normal cone to gph $\mathcal{F}$ at $(\bar{p}, \bar{x}) \in \operatorname{gph} \mathcal{F}$ reduces to

$N((\bar{p}, \bar{x}) ; \operatorname{gph} \mathcal{F})=\left\{\left(p^{*}, x^{*}\right) \in l_{\infty}(T)^{*} \times X^{*} \mid\left\langle\left(p^{*}, x^{*}\right),(p, x)-(\bar{p}, \bar{x})\right\rangle \leq 0\right.$ for $\left.(p, x) \in \operatorname{gph} \mathcal{F}\right\}$

and the coderivative (2.3) of $\mathcal{F}$ admits the representation

$$
D^{*} \mathcal{F}(\bar{p}, \bar{x})\left(x^{*}\right)=\left\{p^{*} \in l_{\infty}(T)^{*} \mid\left\langle p^{*}, \bar{p}\right\rangle-\left\langle x^{*}, \bar{x}\right\rangle=\max _{(p, x) \in \operatorname{gph} \mathcal{F}}\left[\left\langle p^{*}, p\right\rangle-\left\langle x^{*}, x\right\rangle\right]\right\} .
$$

Let us now present two preliminary results that play an important role in our subsequent analysis. The first one is taken from [13, Lemma 2.4] and can be viewed as an extended Farkas lemma for linear infinite inequality systems in Banach spaces. An alternative proof can be derived from [38, Theorem 4].

Lemma 2.1 (extended Farkas lemma). Let $p \in \operatorname{dom} \mathcal{F}$ for the infinite system (1.1) with a Banach decision space $X$, and let $\left(x^{*}, \alpha\right) \in X^{*} \times \mathbb{R}$. The following are equivalent:

(i) We have $\left\langle x^{*}, x\right\rangle \leq \alpha$ whenever $x \in \mathcal{F}(p)$, i.e.,

$$
\left[\left\langle a_{t}^{*}, x\right\rangle \leq b_{t}+p_{t} \text { for all } t \in T\right] \Longrightarrow\left[\left\langle x^{*}, x\right\rangle \leq \alpha\right]
$$


(ii) The pair $\left(x^{*}, \alpha\right)$ satisfies the inclusion

$$
\left(x^{*}, \alpha\right) \in \operatorname{cl}^{*} \operatorname{cone}\left[\left\{\left(a_{t}^{*}, b_{t}+p_{t}\right) \mid t \in T\right\} \cup\{(0,1)\}\right] \text { with } 0 \in X^{*} .
$$

Throughout the paper we largely use the parametric characteristic sets

$$
C(p):=\operatorname{co}\left\{\left(a_{t}^{*}, b_{t}+p_{t}\right) \mid t \in T\right\}, \quad p \in l_{\infty}(T)
$$

and suppose with no loss of generality that our nominal parameter is the zero function $\bar{p}=0$ in the parameter space $l_{\infty}(T)$.

Let us recall a well-recognized qualification condition for linear infinite inequalities, which is often used in problems of semi-infinite and infinite programming.

Definition 2.2 (strong Slater condition). We say that the infinite system (1.1) satisfies the STRONG SLATER CONDition (SSC) at $p=\left(p_{t}\right)_{t \in T}$ if there is $\widehat{x} \in X$ such that

$$
\sup _{t \in T}\left[\left\langle a_{t}^{*}, \widehat{x}\right\rangle-b_{t}-p_{t}\right]<0 .
$$

Furthermore, every point $\widehat{x} \in-X$ satisfying condition (2.9) is a STRONG-SLATER-POINT-for system (1.1) at $p=\left(p_{t}\right)_{t \in T}$.

The next result contains several equivalent descriptions and interpretations of the strong Slater condition used in what follows; the most important is the equivalence (i) $\Longleftrightarrow$ (ii). Note that a similar equivalence can be found in [14] for more general convex systems in locally convex spaces with different spaces of associated parameters. We include here a simplified proof of this equivalence for the reader's convenience.

Lemma 2.3 (equivalent descriptions of the strong Slater condition). Let $X$ be a Banach space, and let $p \in \operatorname{dom} \mathcal{F}$ for the linear infinite inequality system (1.1). Then the following properties are equivalent:

(i) $\mathcal{F}$ satisfies the strong Slater condition at $p$.

(ii) $(0,0) \notin \mathrm{cl}^{*} C(p)$ via the characteristic set from (2.8).

(iii) $p \in \operatorname{int}(\operatorname{dom} \mathcal{F})$.

(iv) $\mathcal{F}$ is Lipschitz-like around $(p, x)$ for all $x \in \mathcal{F}(p)$.

Moreover, if the set $\left\{a_{t}^{*} \mid t \in \mathcal{T}\right\}$ is bounded in $X^{*}$, the conditions above are equivalent to:

(v) there exists $\widehat{x} \in X$ such that $(p, \widehat{x}) \in \operatorname{int}(\operatorname{gph} \mathcal{F})$.

Proof. We begin with the proof of $(\mathrm{i}) \Longrightarrow($ ii). Arguing by contradiction, assume that $(0,0) \in \mathrm{cl}^{*} C(p)$. Then there is a net $\left\{\lambda_{\nu}\right\}_{\nu \in \mathcal{N}} \in \mathbb{R}_{+}^{(T)}$ satisfying $\sum_{t \in T} \lambda_{t \nu}=1$ for all $\nu \in \mathcal{N}$ and the limiting condition

$$
(0,0)=w^{*}-\lim _{\nu} \sum_{t \in T} \lambda_{t \nu}\left(a_{t}^{*}, b_{t}+p_{t}\right)
$$

If $\widehat{x}$ is a strong Slater point for system (1.1) at $p$, we find $\vartheta>0$ such that

$$
\left\langle a_{t}^{*}, \widehat{x}\right\rangle-b_{t}-p_{t} \leq-\vartheta \text { for all } t \in T \text {. }
$$


Then (2.10) leads to the following contradiction:

$$
0=\langle 0, \widehat{x}\rangle+0 \cdot(-1)=\lim _{\nu} \sum_{t \in T} \lambda_{t \nu}\left(\left\langle a_{t}^{*}, \widehat{x}\right\rangle+\left(b_{t}+p_{t}\right) \cdot(-1)\right) \leq-\vartheta
$$

Let us next justify the converse implication (ii) $\Longrightarrow(\mathrm{i})$. By $[13$, Theorem 3.1] we have

$$
p \in \operatorname{dom} \mathcal{F} \Longleftrightarrow(0,-1) \notin \operatorname{cl}^{*} \operatorname{cone}\left\{\left(a_{t}^{*}, b_{t}+p_{t}\right) \mid t \in T\right\} .
$$

Then the strong separation theorem ensures the existence of $(0,0) \neq(v, \alpha) \in X \times \mathbb{R}$ with

$$
\begin{gathered}
\left\langle a_{t}^{*}, v\right\rangle+\alpha\left(b_{t}+p_{t}\right) \leq 0 \text { for all } t \in T \\
\langle 0, v\rangle+(-1) \alpha=-\alpha>0
\end{gathered}
$$

At the same time by (ii) we have $(0,0) \neq(z, \beta) \in X \times \mathbb{R}$ and $\gamma \in \mathbb{R}$ for which

$$
\left\langle a_{t}^{*}, z\right\rangle+\beta\left(b_{t}+p_{t}\right) \leq \gamma<0 \text { whenever } t \in T .
$$

Consider further the combination

$$
(u, \eta):=(z, \beta)+\lambda(v, \alpha)
$$

and select $\lambda>0$ to be sufficiently large to ensure that $\eta<0$. Defining now $\widehat{x}:=-\eta^{-1} u$, we observe from (2.11) and (2.12) that

$$
\left\langle a_{t}^{*}, \widehat{x}\right\rangle-b_{t}-p_{t}=-\eta^{-1}\left(\left\langle a_{t}^{*}, u\right\rangle+\eta\left(b_{t}+p_{t}\right)\right) \leq-\eta^{-1} \gamma<0
$$

This allows us to conclude that $\widehat{x}$ is a strong Slater point for system (1.1) at $p$.

To prove implication (i) $\Longrightarrow$ (iii), assume that $\widehat{x}$ is a strong Slater point for system (1.1) at $p$ and find $\vartheta>0$ such that

$$
\left\langle a_{t}^{*}, \widehat{x}\right\rangle-b_{t}-p_{t} \leq-\vartheta \text { for all } t \in T \text {. }
$$

Then it is obvious that for any $q \in l_{\infty}(T)$ with $\|q\|<\vartheta$ we have $\widehat{x} \in \mathcal{F}(p+q)$. Therefore $p+q \in \operatorname{dom} \mathcal{F}$, and thus (iii) holds.

Let us further proceed with justifying implication (iii) $\Longrightarrow($ i). If $p \in \operatorname{int}(\operatorname{dom} \mathcal{F})$, then $p+q \in \operatorname{dom} \mathcal{F}$ provided that $q_{t}=-\vartheta$ as $t \in T$ and that $\vartheta>0$ is sufficiently small. Thus every $\widehat{x} \in \mathcal{F}(p+q)$ is a strong Slater point for the infinite system (1.1) at $p$.

The equivalence between (iii) and (iv) is a consequence of the classical Robinson-Ursescu closed graph/metric regularity theorem; see, e.g., [20] and [31, Chapter 4] with more discussions and references therein.

It remains to consider condition ( $v$ ). We can easily observe that (v) always implies (iii) and so the other conditions of the lemma. Suppose now that that the set $\left\{a_{t}^{*} \mid t \in T\right\}$ is bounded in $X^{*}$ and show that in this case (i) implies (v). Select $M \geq 0$ such that $\left\|a_{t}^{*}\right\| \leq M$ for every $t \in T$ and take $\widehat{x} \in X$ satisfying (2.9). Denote

$$
\gamma:=-\sup _{t \in T}\left[\left\langle a_{t}^{*}, \widehat{x}\right\rangle-b_{t}-p_{t}\right]>0
$$


and consider any pair $\left(p^{\prime}, u\right) \in l_{\infty}(T) \times X$ satisfying the relationships

$$
\|u\| \leq \eta:=\gamma /(M+1)>0 \text { and }\left\|p^{\prime}\right\| \leq \eta \text {. }
$$

It is easy to see that for such $\left(p^{\prime}, u\right)$ and every $t \in T$ we have

$$
\left\langle a_{t}^{*}, \widehat{x}+u\right\rangle-b_{t}-p_{t}-p_{t}^{\prime} \leq-\gamma+M\|u\|+\left\|p^{\prime}\right\| \leq \eta(M+1)-\gamma=0,
$$

and so $\left(p+p^{\prime}, \widehat{x}+u\right) \in \operatorname{gph} \mathcal{F}$. Thus $(p, \widehat{x}) \in \operatorname{int}(\operatorname{gph} \mathcal{F})$, which gives $(\mathrm{i}) \Longrightarrow(\mathrm{v})$ and completes the proof of the lemma.

Remark 2.4 (relationships between interiority and boundedness condition). Note that we have the nonempty interiority condition $\operatorname{int}(\operatorname{gph} \mathcal{F}) \neq \emptyset$ provided that the set $\left\{a_{t}^{*} \mid t \in T\right\}$ is bounded in $X^{*}$ and that $\operatorname{gph} \mathcal{F} \neq \emptyset$. Also we have $\operatorname{int}(\operatorname{dom} \mathcal{F}) \neq \emptyset$ if $\operatorname{gph} \mathcal{F} \neq \emptyset$ with no boundedness assumption.

To justify the first statement, take $(p, x) \in \operatorname{gph} \mathcal{F}$ and $\left\|a_{t}^{*}\right\| \leq M<\infty$ for every $t \in T$ and define $e \in l_{\infty}(T)$ by $e_{t}:=1$ for all $t \in T$. Then arguing similarly to the proof of the last implication in Lemma 2.1, we get $\left(p+e+p^{\prime}, x+u\right) \in \operatorname{gph} \mathcal{F}$ whenever $\left\|p^{\prime}\right\| \leq \eta:=1 /(M+1)$ and $\|u\| \leq \eta$. This gives $(p+e, x) \in \operatorname{int}(\operatorname{gph} \mathcal{F})$. If furthermore $(p, x) \in \operatorname{gph} \mathcal{F}$, we can easily check that $x \in \mathcal{F}\left(p+e+p^{\prime}\right)$ provided that $\left\|p^{\prime}\right\| \leq 1$, and therefore $p+e \in \operatorname{int}(\operatorname{dom} \mathcal{F})$.

The major space for our consideration in this paper is the parameter space $l_{\infty}(T)$ of bounded functions $p: T \rightarrow \mathbb{R}$ on $T$ with the supremum norm (1.2). It is obviously a Banach space that is never finite-dimensional when the index set $T$ is infinite, which is our standing assumption. Let us show that it is never Asplund.

Proposition 2.5 (parameter space is never Asplund). The parameter space $l_{\infty}(T)$ is Asplund if and only if the index set $T$ is finite.

Proof. If $T$ is countable (i.e., $T=\mathbb{N}$ and the parameter space is the classical space of sequences $\left.l_{\infty}\right)$, the proof can be found in [34, Example 1.21]; in fact, this space is not even weak Asplund. The same arguments can be adapted for any infinite index set $T$.

Finally in this section, we recall a convenient description of the topological dual space $l_{\infty}(T)^{*}$ to the parameter space $l_{\infty}(T)$. According to [16], there is an isometric isomorphism between $l_{\infty}(T)^{*}$ and the space of bounded and additive measures

$$
b a(T)=\left\{\mu: 2^{T} \rightarrow \mathbb{R} \mid \mu \text { is bounded and additive }\right\}
$$

satisfying the relationship

$$
\langle\mu, p\rangle=\int_{T} p_{t} \mu(d t) \text { with } p=\left(p_{t}\right)_{t \in T} .
$$

The dual norm on $b a(T)$ corresponding to (1.2) is the total variation of $\mu \in b a(T)$ on the index set $T$ defined by

$$
\|\mu\|:=\sup _{A \subset T} \mu(A)-\inf _{B \subset T} \mu(B) .
$$

In what follows we always identify the measure space $b a(T)$ with the dual parameter space $l_{\infty}(T)^{*}$ and use, for the notational unification, $p^{*} \in l_{\infty}(T)^{*}$ instead of $\mu \in b a(T)$. 


\section{Computing Coderivatives and Coderivative Norms for Lin- ear Infinite Inequality Systems}

In this section we establish a constructive representation of the coderivative $D^{*} \mathcal{F}(0, \bar{x})$ for the feasible solution map $\mathcal{F}$ at the nominal point $(0, \bar{x})$ and compute its norm $\left\|D^{*} \mathcal{F}(0, \bar{x})\right\|$ in terms of the initial data of the linear infinite inequality system (1.1). Let us first describe the normal cone to the convex graph (2.6) employing the extended Farkas lemma presented above. In what follows $\delta_{t}$ denotes the classical Dirac measure at $t \in T$ satisfying

$$
\left\langle\delta_{t}, p\right\rangle=p_{t} \text { as } t \in T \text { for } p=\left(p_{t}\right)_{t \in T} \in l_{\infty}(T) .
$$

Proposition 3.1 (computing normals to the graphical set of feasible solutions). Let $(\bar{p}, \bar{x}) \in \operatorname{gph} \mathcal{F}$ for the graphical set (2.6) with a Banach decision space $X$, and let $\left(p^{*}, x^{*}\right) \in l_{\infty}(T)^{*} \times X^{*}$. Then we have $\left(p^{*}, x^{*}\right) \in N((\bar{p}, \bar{x}) ; \operatorname{gph} \mathcal{F})$ if and only if

$$
\left(p^{*}, x^{*},\left\langle p^{*}, \bar{p}\right\rangle+\left\langle x^{*}, \bar{x}\right\rangle\right) \in \mathrm{cl}^{*} \operatorname{cone}\left[\left\{\left(-\delta_{t}, a_{t}^{*}, b_{t}\right) \mid t \in T\right\} \cup\{(0,0,1)\}\right],
$$

where $0 \in l_{\infty}(T)^{*}$ and $0 \in X^{*}$ stand for the first and second entry of the last triple in (3.1), respectively. Furthermore, the inclusion $\left(\bar{p}^{*}, \bar{x}^{*}\right) \in N((\bar{p}, \bar{x}) ; \overline{g h} \mathcal{F})$ implies that $\bar{p}^{*} \leq 0$ in the space ba $(\mathcal{T})$, i.e., $p^{*}(A) \leq 0$ for all $A \subset T$.

Proof. Observe from (2.6) and from the definition of the Dirac measure that the graph of $\mathcal{F}$ admits the representation

$$
\operatorname{gph} \mathcal{F}=\left\{(p, x) \in l_{\infty}(T) \times X \mid\left\langle a_{t}^{*}, x\right\rangle-\left\langle\delta_{t}, p\right\rangle \leq b_{t} \text { for all } t \in T\right\} .
$$

Therefore we have $\left(p^{*}, x^{*}\right) \in N((\bar{p}, \bar{x}) ; \operatorname{gph} \mathcal{F})$ if and only if

$$
\left\langle p^{*}, p\right\rangle+\left\langle x^{*}, x\right\rangle \leq\left\langle p^{*}, \bar{p}\right\rangle+\left\langle x^{*}, \bar{x}\right\rangle
$$

for every $(p, x) \in \operatorname{gph} \mathcal{F}$. Employing now the equivalence between (i) and (ii) in Lemma 2.1, we conclude that $\left(p^{*}, x^{*}\right) \in N((\bar{p}, \bar{x}) ; \mathrm{gph} \mathcal{F})$ if and only if inclusion (3.1) holds.

To justify the last statement of the proposition, for every set $A \subset T$ consider its characteristic function $\chi_{A}: T \rightarrow\{0,1\}$ defined by

$$
\chi_{A}(t):= \begin{cases}1, & \text { if } t \in A, \\ 0, & \text { if } t \notin A .\end{cases}
$$

It is obvious that the inclusion $(p, x) \in \operatorname{gph} \mathcal{F}$ implies that $\left(p+\lambda \chi_{A}, x\right) \in \operatorname{gph} \mathcal{F}$ for each $\lambda>0$. Replacing now in (3.2) the pair $(p, x)$ by $\left(p+\lambda \chi_{A}, x\right)$, keeping $p$ and $x$ fixed, dividing both sides of the inequality by $\lambda$ and letting $\lambda \rightarrow \infty$, we get the relationships

$$
\left\langle p^{*}, \chi_{A}\right\rangle=\int_{T} \chi_{A}(t) p^{*}(d t)=p^{*}(A) \leq 0,
$$

which complete the proof of the proposition.

Based on the above proposition and the general coderivative definition, we now obtain a constructive representation of the coderivative $D^{*} \mathcal{F}(0, \bar{x})$ in question. 
Theorem 3.2 (coderivative of the feasible solution map). Let $\bar{x} \in \mathcal{F}(0)$ for the feasible solution map $\mathcal{F}: l_{\infty}(T) \rightrightarrows X$ from (1.1) with a Banach decision space $X$. Then $p^{*} \in D^{*} \mathcal{F}(0, \bar{x})\left(x^{*}\right)$ if and only if

$$
\left(p^{*},-x^{*},-\left\langle x^{*}, \bar{x}\right\rangle\right) \in \mathrm{cl}^{*} \operatorname{cone}\left\{\left(-\delta_{t}, a_{t}^{*}, b_{t}\right) \mid t \in T\right\} .
$$

Proof. By the coderivative construction (2.3) applied to $\mathcal{F}$ and by the normal cone formula from Proposition 3.1 as $\bar{p}=0$ we get that $p^{*} \in D^{*} \mathcal{F}(0, \bar{x})\left(x^{*}\right)$ if and only if

$$
\left(p^{*},-x^{*},-\left\langle x^{*}, \bar{x}\right\rangle\right) \in \mathrm{cl}^{*} \operatorname{cone}\left[\left\{\left(-\delta_{t}, a_{t}^{*}, b_{t}\right) \mid t \in T\right\} \cup\{(0,0,1)\}\right] .
$$

To justify the coderivative representation claimed in the theorem, we need to show that inclusion (3.4) implies in fact the "smaller" one in (3.3). Assuming indeed that (3.4) holds, we find by the structure of the right-hand side on (3.4) some nets $\left\{\lambda_{\nu}\right\}_{\nu \in \mathcal{N}} \subset \mathbb{R}_{+}^{(T)}$ and $\left\{\gamma_{\nu}\right\}_{\nu \in \mathcal{N}} \subset \mathbb{R}_{+}$satisfying the limiting relationship

$$
\left(p^{*},-x^{*},-\left\langle x^{*}, \bar{x}\right\rangle\right)=w^{*}-\lim _{\nu}\left(\sum_{t \in T} \lambda_{t \nu}\left(-\delta_{t}, a_{t}^{*}, b_{t}\right)+\gamma_{\nu}(0,0,1)\right)
$$

where $\lambda_{t \nu}$ stands for the $t$-entry of $\lambda_{\nu}=\left(\lambda_{t \nu}\right)_{t \in T}$ as $\nu \in \mathcal{N}$. It follows directly from the component structure in (3.5) that

$$
0=\left\langle p^{*}, 0\right\rangle+\left\langle-x^{*}, \bar{x}\right\rangle+\left(-\left\langle x^{*}, \bar{x}\right\rangle\right)(-1)=\lim _{\nu}\left(\sum_{t \in T} \lambda_{t \nu}\left(\left\langle a_{t}^{*}, \bar{x}\right\rangle-b_{t}\right)-\gamma_{\nu}\right) .
$$

Taking finally into account the definition of the positive cone $\mathbb{R}_{+}^{(T)}$ and the fact that the pair $(0, \bar{x})$ satisfies the infinite inequality system in (1.1), we conclude from (3.6) that $\lim _{\nu} \gamma_{\nu}=0$. This justifies (3.3) and thus completes the proof of the theorem.

The next consequence of Theorem 3.2 is useful in what follows.

Corollary 3.3 (limiting descriptions of coderivatives). If $p^{*} \in D^{*} \mathcal{F}(0, \bar{x})\left(x^{*}\right)$ in the framework of Theorem 3.2 , then there is a net $\left\{\lambda_{\nu}\right\}_{\nu \in \mathcal{N}} \subset \mathbb{R}_{+}^{(T)}$ such that

$$
\begin{gathered}
\sum_{t \in T} \lambda_{t \nu} \rightarrow\left\|p^{*}\right\|=-\left\langle p^{*}, e\right\rangle, \\
\sum_{t \in T} \lambda_{t \nu} a_{t}^{*} \stackrel{w^{*}}{\longrightarrow}-x^{*}, \text { and } \sum_{t \in T} \lambda_{t \nu} b_{t} \rightarrow-\left\langle x^{*}, \bar{x}\right\rangle .
\end{gathered}
$$

Proof. It follows from Theorem 3.2 that there is a net $\left\{\lambda_{\nu}\right\}_{\nu \in \mathcal{N}} \subset \mathbb{R}_{+}^{(T)}$ such that

$$
\sum_{t \in T} \lambda_{t \nu} \delta_{t} \stackrel{w^{*}}{\longrightarrow}-p^{*}, \quad \sum_{t \in T} \lambda_{t \nu} a_{t}^{*} \stackrel{w^{*}}{\longrightarrow}-x^{*}, \text { and } \sum_{t \in T} \lambda_{t \nu} b_{t} \rightarrow-\left\langle x^{*}, \bar{x}\right\rangle,
$$

which readily imply the relationships

$$
\left\langle\sum_{t \in T} \lambda_{t \nu} \delta_{t}, e\right\rangle=\sum_{t \in T} \lambda_{t \nu} \rightarrow\left\langle p^{*},-e\right\rangle=: \lambda \in[0, \infty) .
$$


Since the dual norm on $X^{*}$ is $w^{*}$-lower semicontinuous, we have

$$
\left\|p^{*}\right\| \leq \liminf _{\nu \in \mathcal{N}}\left\|\sum_{t \in T} \lambda_{t \nu} \delta_{t}\right\| \leq \liminf _{\nu \in \mathcal{N}} \sum_{t \in T} \lambda_{t \nu}=\lambda .
$$

It follows at the same time from the norm definition that

$$
\left\|p^{*}\right\|=\sup _{\|p\| \leq 1}\left\langle p^{*}, p\right\rangle \geq\left\langle p^{*},-e\right\rangle=\lambda
$$

which finally yields $\left\|p^{*}\right\|=-\left\langle p^{*}, e\right\rangle$ and completes the proof of the corollary.

Our further intention is to provide the exact calculation of the coderivative norm

$$
\left\|D^{*} \mathcal{F}(0, \bar{x})\right\|:=\sup \left\{\left\|p^{*}\right\| \mid p^{*} \in D^{*} \mathcal{F}(0, \bar{x})\left(x^{*}\right),\left\|x^{*}\right\| \leq 1\right\}
$$

in terms of the initial data of the linear infinite inequality system (1.1). A part of our analysis in this direction is the following lemma on properties of the characteristic set (2.8) at $p=0$, which is also used in Section 4 to compute the exact Lipschitzian bound lip $\mathcal{F}(0, \bar{x})$.

Lemma 3.4 (properties of the characteristic set). Let $X$ be an arbitrary Banach space. The following assertions hold:

(i) Assume that $\bar{x} \in \mathcal{F}(0)$ is not a strong Slater point for the infinite system (1.1) at $p=0$ and that the collection $\left\{a_{t}^{*} \mid t \in T\right\}$ is bounded in $X^{*}$. Then the set

$$
S:=\left\{x^{*} \in X^{*} \mid\left(x^{*},\left\langle x^{*}, \vec{x}\right\rangle\right) \in \mathrm{cl}^{*} C(0)\right\}
$$

built upon the characteristic set $C(0)$ in (2.8) is nonempty and $w^{*}$-compact at $X^{*}$.

(ii) Assume that $\bar{x} \in \mathcal{F}(0)$ is a strong Slater point of (1.1) at $p=0$. Then the set $S$ in (3.8) is empty.

Proof. To justify (i), let $\vec{x}$ be not a strong Slater point for the infinite system (1.1) at $p=0$. Then there is a sequence $\left\{t_{k}\right\}_{k \in \mathbb{N}} \subset T$ such that $\lim _{k}\left(\left\langle a_{t_{k}}^{*}, \bar{x}\right\rangle-b_{t_{k}}\right)=0$. The boundedness of $\left\{a_{t}^{*} \mid t \in T\right\}$ implies by the classical Alaoglu-Bourbaki theorem that this set is relatively $w^{*}$-compact in $X^{*}$, i.e., there is a subnet $\left\{a_{t_{\nu}}^{*}\right\}_{\nu \in \mathcal{N}}$ of the latter sequence that $w^{*}$-converges to some element $u^{*} \in \mathrm{cl}^{*}\left\{a_{t}^{*} \mid t \in T\right\}$. This gives $\lim _{\nu} b_{t_{\nu}}=\left\langle u^{*}, \tilde{x}\right\rangle$ and therefore

$$
\left(u^{*},\left\langle u^{*}, \bar{x}\right\rangle\right)=w^{*}-\lim _{\nu}\left(a_{t_{\nu}}^{*}, b_{t_{\nu}}\right) \in \mathrm{cl}^{*} C(0),
$$

which justifies the nonemptiness of the set $S$ in (3.8). Next we prove that $S$ is $w^{*}$-compact.

Indeed, by our assumption the set $A:=\left\{a_{t}^{*} \mid t \in T\right\}$ is bounded in $X^{*}$, and so is $\mathrm{cl}^{*} \operatorname{co} A$; the latter is actually $w^{*}$-compact due to its automatic $w^{*}$-closedness. Observe further that the set $S$ in (3.8) is a preimage of $\mathrm{cl}^{*} C(0)$ under the $w^{*}$-continuous mapping $x^{*} \mapsto\left(x^{*},\left\langle x^{*}, \bar{x}\right\rangle\right)$, and thus it is $w^{*}$-closed in $X^{*}$. Since $S$ is a subset of $\mathrm{cl}^{*} \operatorname{co} A$, it is also bounded and hence $w^{*}$-compact in $X^{*}$. This completes the proof of assertion (i).

To prove assertion (ii), let $\vec{x}$ be a strong Slater point of system (1.1) at $p=0$, and let

$$
\gamma:=-\sup _{t \in T}\left\{\left\langle a_{t}^{*}, \bar{x}\right\rangle-b_{t}\right\}
$$


Then we have the inequality

$$
\left\langle x^{*}, \bar{x}\right\rangle \leq \beta-\gamma \text { whenever }\left(x^{*}, \beta\right) \in \mathrm{cl}^{*} C(0),
$$

which justifies (ii) and thus completes the proof of the lemma.

Now we are ready to compute the coderivative norm $\left\|D^{*} \mathcal{F}(0, \bar{x})\right\|$ at the reference point.

Theorem 3.5 (computing the coderivative norm). Let $\bar{x} \in \operatorname{dom} \mathcal{F}$ for the infinite system (1.1) with an arbitrary Banach space $X$ of decision variables. Assume that $\mathcal{F}$ satisfies the strong Slater condition at $p=0$ and that the coefficient set $\left\{a_{t}^{*} \mid t \in T\right\}$ is bounded in $X^{*}$. The following assertions hold:

(i) If $\bar{x}$ is a strong Slater point for $\mathcal{F}$ at $p=0$, then $\left\|D^{*} \mathcal{F}(0, \bar{x})\right\|=0$.

(ii) If $\bar{x}$ is not a strong Slater point for $\mathcal{F}$ at $p=0$, then the coderivative norm (3.7) is positive and is computed by

$$
\left\|D^{*} \mathcal{F}(0, \vec{x})\right\|=\max \left\{\left\|x^{*}\right\|^{-1} \mid\left(x^{*},\left\langle x^{*}, \vec{x}\right\rangle\right) \in \mathrm{cl}^{*} C(0)\right\}
$$

via the $w^{*}$-closure of the characteristic set $(2.8)$ at $p=0$.

Proof. To justify assertion (i), assume that $\bar{x}$ is a strong Slater point for the system $\mathcal{F}$ at $p=0$. It follows from the proof of implication $(\mathrm{i}) \Longrightarrow(\mathrm{v})$ in Lemma 2.3 that we have $(0, \tilde{x}) \in \operatorname{int}(\operatorname{gph} \mathcal{F})$. It gives

$$
N((0, \bar{x}) ; \operatorname{gph} \mathcal{F})=\{(0,0)\},
$$

and the conclusion in (i) follows from (2.3) and (3.7).

To prove assertion (ii), take $x^{*} \in X^{*}$ such that $\left(x^{*},\left\langle x^{*}, \bar{x}\right\rangle\right) \in \mathrm{cl}^{*} C(0)$; the latter set is nonempty according to Lemma 3.4. Then there exists a net $\left\{\lambda_{\nu}\right\}_{\nu \in \mathcal{N}} \subset \mathbb{R}_{+}^{(T)}$ with $\sum_{t \in T} \lambda_{t \nu}=1$ for all $\nu \in \mathcal{N}$ such that

$$
\sum_{t \in T} \lambda_{t \nu} a_{t}^{*} \stackrel{w^{*}}{\rightarrow} x^{*} \text { and } \sum_{t \in T} \lambda_{t \nu} b_{t} \rightarrow\left\langle x^{*}, \bar{x}\right\rangle .
$$

Form further net elements $p_{\nu}^{*} \in l_{\infty}(T)^{*}$ by

$$
p_{\nu}^{*}:=-\sum_{t \in T} \lambda_{t \nu} \delta_{t}, \quad \nu \in \mathcal{N} .
$$

Since $\left\|p_{\nu}^{*}\right\|=\left\langle p_{\nu}^{*},-e\right\rangle=1$, the Alaoglu-Bourbaki theorem allows us to select a subnet (without relabeling) such that $p_{\nu}^{*} \stackrel{w^{*}}{\longrightarrow} p^{*}$ with $\left\|p^{*}\right\| \leq 1$. Following the same reasoning as in the proof of Corollary 3.3, we conclude that

$$
1=\lim _{\nu \in \mathcal{N}} \sum_{t \in T} \lambda_{t \nu}=\left\|p^{*}\right\|=\left\langle p^{*},-e\right\rangle
$$

Moreover, we also obtain the inclusion

$$
\left(p^{*}, x^{*},\left\langle x^{*}, \bar{x}\right\rangle\right) \in \mathrm{cl}^{*} \operatorname{co}\left\{\left(-\delta_{t}, a_{t}^{*}, b_{t}\right) \mid t \in T\right\}
$$


which implies by Theorem 3.2 that

$$
p^{*} \in D^{*} \mathcal{F}(0, \ddot{x})\left(-x^{*}\right) .
$$

Suppose now that $x^{*}=0$ in (3.11). Since $p^{*} \neq 0$ by (3.10), we get from (3.11) that

$$
D^{*} \mathcal{F}(0, \bar{x})(0) \neq\{0\},
$$

which yields by [31, Theorem 1.44] that $\mathcal{F}$ is not Lipschitz-like around $(0, \bar{x})$, and therefore it cannot satisfy the strong Slater condition by implication (i) $\Longrightarrow$ (iv) in Lemma 2.3. This contradicts the assumption of the theorem.

Thus $x^{*} \neq 0$ in (3.11), and we derive from the latter relationship that

$$
\left\|x^{*}\right\|^{-1} p^{*} \in D^{*} \mathcal{F}(0, \bar{x})\left(-\left\|x^{*}\right\|^{-1} x^{*}\right)
$$

which gives in turn that

$$
\left\|D^{*} \mathcal{F}(0, \bar{x})\right\| \geq\|\| x^{*}\left\|^{-1} p^{*}\right\|=\left\|x^{*}\right\|^{-1}
$$

and-hence-justifies-the-inequality " $\geq$ " in (3.9).

It remains to prove the opposite inequality in (3.9). For the nonempty and $w^{*}$-compact set $S$ in (3.8) we have $0 \notin S$ by Lemma 2.3 , which ensures the $w^{*}$-upper semicontinuity of the function $x^{*} \mapsto\left\|x^{*}\right\|^{-1}$ on $S$. Thus the supremum in the right-hand side of (3.9) is attained and belongs to $(0, \infty)$. Then condition (v) in Lemma 2.3 implies that $(0, \widehat{x}) \in \operatorname{int}(\operatorname{gph} \mathcal{F})$ for some $\widehat{x} \in X$, and so $0 \in \operatorname{int}(\operatorname{dom} \mathcal{F})$. Moreover, we have that $p^{*} \in D^{*} \mathcal{F}(0, \bar{x})\left(-x^{*}\right)$ if and only if $\left(p^{*}, x^{*}\right) \in N((0, \bar{x}) ; \operatorname{gph} \mathcal{F})$, which is equivalent to

$$
\left\langle p^{*}, p\right\rangle+\left\langle x^{*}, x\right\rangle \leq\left\langle x^{*}, \bar{x}\right\rangle \text { for all }(p, x) \in \operatorname{gph} \mathcal{F} \text {. }
$$

This allows us, by taking into account that $0 \in \operatorname{int}(\operatorname{dom} \mathcal{F})$, to arrive at the equivalences

$$
p^{*} \in D^{*} \mathcal{F}(0, \bar{x})(0) \Longleftrightarrow\left\langle p^{*}, p\right\rangle \leq 0 \text { for all } p \in \operatorname{dom} \mathcal{F} \Longleftrightarrow p^{*}=0 .
$$

Observe furthermore that, since $\bar{x}$ is not a strong Slater point for $\mathcal{F}$ at $p=0$, we have $(0, \bar{x}) \notin \operatorname{int}(\operatorname{gph} \mathcal{F})$ and thus conclude by the classical separation theorem that there is a pair $\left(p^{*}, x^{*}\right) \neq(0,0)$ for which relations (3.13) holds. Employing (3.14), we have $x^{*} \neq 0$ and $p^{*} \in D^{*} \mathcal{F}(0, \bar{x})\left(-x^{*}\right)$.

Take now $p^{*} \in D^{*} \mathcal{F}(0, \bar{x})\left(-x^{*}\right)$ with $\left\|x^{*}\right\| \leq 1$ and suppose that $x^{*} \neq 0$; the arguments of the previous paragraph ensure the existence of such an element. By Corollary 3.3 there is a net $\left\{\lambda_{\nu}\right\}_{\nu \in \mathcal{N}} \subset \mathbb{R}_{+}^{(T)}$ for which

$$
\begin{aligned}
& \gamma_{\nu}:=\sum_{t \in T} \lambda_{t \nu} \rightarrow\left\|p^{*}\right\|=-\left\langle p^{*}, e\right\rangle \\
& x_{\nu}^{*}:=\sum_{t \in T} \lambda_{t \nu} a_{t}^{*} \stackrel{w^{*}}{\longrightarrow} x^{*} \text {, and } \sum_{t \in T} \lambda_{t \nu} b_{t} \rightarrow\left\langle x^{*}, \bar{x}\right\rangle .
\end{aligned}
$$

Taking $M \geq\left\|a_{t}^{*}\right\|$ for every $t \in T$, we get the estimate

$$
\left\|x_{\nu}^{*}\right\| \leq M \gamma_{\nu} \quad \text { whenever } \quad \nu \in \mathcal{N}
$$


and also the limiting relationships

$$
0<\left\|x^{*}\right\| \leq \liminf _{\nu \in \mathcal{N}}\left\|x_{\nu}^{*}\right\| \leq M \liminf _{\nu \in \mathcal{N}} \gamma_{\nu}=M\left\|p^{*}\right\|,
$$

which ensure that $p^{*} \neq 0$. It follows furthermore that

$$
\left\|p^{*}\right\|^{-1}\left(x^{*},\left\langle x^{*}, \bar{x}\right\rangle\right) \in \mathrm{cl}^{*} C(0) .
$$

Remembering finally that $0<\left\|x^{*}\right\| \leq 1$, we arrive at the estimates

$$
\left\|p^{*}\right\| \leq\|\| p^{*}\left\|^{-1} x^{*}\right\|^{-1} \leq \max \left\{\left\|u^{*}\right\|^{-1} \mid\left(u^{*},\left\langle u^{*}, \bar{x}\right\rangle\right) \in \operatorname{cl}^{*} C(0)\right\},
$$

which justify the inequality " $\leq$ " in (3.9) and complete the proof of the theorem.

\section{Characterizations of Robust Lipschitzian Stability for Fea- sible Solution Maps}

In this section we employ the above coderivative analysis combined with appropriate techniques developed in linear semi-infinite/infinite programming to establish a coderivative characterization of robust Lipschitzian stability, in the sense discussed in Section 2, for the infinite inequality system $\mathcal{F}$ in (1.1) at the reference point $(0, \bar{x})$ with computing the exact Lipschitzian bound lip $\mathcal{F}(0, \bar{x})$.

The first result of this section establishes the coderivative necessary and sufficient condition in form (2.4) for the Lipschitz-like property of $\mathcal{F}$ around $(0, \bar{x}) \in \operatorname{gph} \mathcal{F}$ in the general setting under consideration.

Theorem 4.1 (coderivative criterion for robust Lipschitzian stability of linear infinite inequalities). Let $\bar{x} \in \mathcal{F}(0)$ for the infinite inequality system (1.1) with a Banach space $X$ of decision variables. Then $\mathcal{F}$ is Lipschitz-like around $(0, \bar{x})$ if and only if

$$
D^{*} \mathcal{F}(0, \bar{x})(0)=\{0\} .
$$

Proof. The "only if" part follows from [31, Theorem 1.44] specified for the mapping $\mathcal{F}: l_{\infty}(T) \rightrightarrows X$ under consideration. Let us now prove the "if" part of the theorem.

Arguing by contradiction, suppose that $D^{*} \mathcal{F}(0, \bar{x})(0)=\{0\}$ while the mapping $\mathcal{F}$ is not Lipschitz-like around $(0, \bar{x})$. Then, by the equivalence between properties (ii) and (iv) of Lemma 2.3, we get the inclusion

$$
(0,0) \in \operatorname{cl}^{*} \operatorname{co}\left\{\left(a_{t}^{*}, b_{t}\right) \in X^{*} \times \mathbb{R} \mid t \in T\right\},
$$

which means that there is a net $\left\{\lambda_{\nu}\right\}_{\nu \in \mathcal{N}} \in \mathbb{R}_{+}^{(T)}$ such that $\sum_{t \in T} \lambda_{t \nu}=1$ for all $\nu \in \mathcal{N}$ and

$$
w^{*}-\lim _{\nu} \sum_{t \in T} \lambda_{t \nu}\left(a_{t}^{*}, b_{t}\right)=(0,0) .
$$


Since the net $\left\{\sum_{t \in T} \lambda_{t \nu}\left(-\delta_{t}\right)\right\}_{\nu \in \mathcal{N}}$ is obviously bounded in $l_{\infty}(T)^{*}$, the Alaoglu-Bourbaki theorem ensures the existence of its subnet (with no relabeling) that $w^{*}$-converges to some element $p^{*} \in l_{\infty}(T)^{*}$, i.e.,

$$
p^{*}=w^{*}-\lim _{\nu} \sum_{t \in T} \lambda_{t \nu}\left(-\delta_{t}\right) .
$$

It follows from (4.3) by the Dirac function definition that

$$
\left\langle p^{*},-e\right\rangle=\lim _{\nu \in \mathcal{N}} \sum_{t \in T} \lambda_{t \nu}=1, \text { where } e=\left(e_{t}\right)_{t \in T} \text { with } e_{t}=1 \text { for all } t \in T,
$$

which implies that $p^{*} \neq 0$. It follows from (4.2) and (4.3) that

$$
\left(p^{*}, 0,0\right)=w^{*}-\lim _{\nu} \sum_{t \in T} \lambda_{t \nu}\left(-\delta_{t}, a_{t}^{*}, b_{t}\right) \text { with } p^{*} \neq 0
$$

and therefore, by the explicit coderivative description of Theorem 3.2, we get the inclusion $p^{*} \in D^{*} \mathcal{F}(0, \bar{x})(0) \backslash\{0\}$, which contradicts the assumed condition (4.1). This justifies the sufficiency of the coderivative condition (4.1) for the Lipschitz-like property of $\mathcal{F}$ around $(0, \bar{x})$ and thus completes the proof of the theorem.

Our further goal_is_to compute the exact Lipschitzian bound lip $\mathcal{F}(0, \vec{x})$ of $\mathcal{F}$ around $(0, \bar{x})$. We are going to do it on the base of the distance representation (2.2) for the exact Lipschitzian bound. To proceed, denote

$$
H\left(x^{*}, \alpha\right):=\left\{x \in X \mid\left\langle x^{*}, x\right\rangle \leq \alpha\right\} \text { for }\left(x^{*}, \alpha\right) \in X^{*} \times \mathbb{R}
$$

and observe the following representation (known as the Ascoli formula; see, e.g., [2]):

$$
\operatorname{dist}\left(x ; H\left(x^{*}, \alpha\right)\right)=\frac{\left[\left\langle x^{*}, x\right\rangle-\alpha\right]_{+}}{\left\|x^{*}\right\|},
$$

where $[\gamma]_{+}:=\max \{\gamma, 0\}$ for $\gamma \in \mathbb{R}$. Recall that we are under the convention $0 / 0:=0$.

The next proposition and the subsequent lemma, which are certainly of their own interest, provide a significant extension of the Ascoli formula (4.4) to the case of infinite systems of linear inequalities instead of the single one as in (4.4). These results are essentially employed in what follows for computing the exact Lipschitzian bound $\operatorname{lip} \mathcal{F}(0, \bar{x})$. We refer the reader to [6, Lemma 2.3] and [7, Lemma 1] for related results in the framework of semi-infinite programming and observe that in infinite dimensions we use the $w^{*}$-closure of the characteristic sets $C(p)$ from (2.8); see also Example 4.4 below for more discussions.

From now on, given an extended-real-valued function $f: X \rightarrow \overline{\mathbb{R}}:=(-\infty, \infty]$ assumed to be proper (i.e., not identically equal $\infty$ ), consider its Fenchel conjugate $f^{*}: X^{*} \rightarrow \overline{\mathbb{R}}$ defined by

$$
f^{*}\left(x^{*}\right):=\sup \left\{\left\langle x^{*}, x\right\rangle-f(x) \mid x \in X\right\}=\sup \left\{\left\langle x^{*}, x\right\rangle-f(x) \mid x \in \operatorname{dom} f\right\},
$$

where $\operatorname{dom} f:=\{x \in X \mid f(x)<\infty\}$ is the effective domain of $f$. In what follows we also use the standard notation epi $f$ for the epigraph of $f$ given by

$$
\text { epi } f:=\{(x, \gamma) \in X \times \mathbb{R} \mid x \in \operatorname{dom} f, f(x) \leq \gamma\} .
$$

The following result gives an exact formula for computing the distance to a set defined by a convex inequality via the corresponding conjugate function. 
Proposition 4.2 (distance function for solutions of convex inequalities in Banach spaces). Let $g: X \rightarrow \overline{\mathbb{R}}$ be a proper convex function on a Banach space $X$, and let

$$
Q:=\{y \in X \mid g(y) \leq 0\} .
$$

Assume the fulfillment of the classical Slater condition: there is $\widehat{x} \in X$ such that $g(\widehat{x})<0$. Then the distance function to the set $Q$ in (4.6) is computed by

$$
\operatorname{dist}(x ; Q)=\max _{\left(x^{*}, \alpha\right) \in \operatorname{epi} g^{*}} \frac{\left[\left\langle x^{*}, x\right\rangle-\alpha\right]_{+}}{\left\|x^{*}\right\|} .
$$

Proof. Observe that the nonemptiness of $Q$ in (4.6) yields that $\alpha \geq 0$ whenever $(0, \alpha) \epsilon$ epi $g^{*}$ and that the possibility of $x^{*}=0$ is not an obstacle in (4.7) under our convention $0 / 0=0$. Obviously the distance function $\operatorname{dist}(x ; Q)$ is nothing else but the optimal value function in the parametric convex optimization problem

$$
\text { minimize }\|y-x\| \text { subject to } g(y) \leq 0 \text {. }
$$

Since the classical_Slater condition holds for problem (4.8) by our assumption, we have the strong Lagrange duality in (4.8) by, e.g., [39, Theorem 2.9.3], which gives

$$
\begin{aligned}
\operatorname{dist}(x ; Q) & =\max _{\lambda \geq 0} \inf _{y \in X}\{\|y-x\|+\lambda g(y)\} \\
& =\max \left\{\max _{\lambda>0} \inf _{y \in X}\{\|y-x\|+\lambda g(y)\}, \inf _{y \in X}\|y-x\|\right\} \\
& =\max \left\{\max _{\lambda>0} \inf _{y \in X}\{\|y-x\|+\lambda g(y)\}, 0\right\} .
\end{aligned}
$$

Applying the classical Fenchel duality theorem to the inner infimum problem above for a fixed $\lambda>0$ (observing to this end that Rockafellar's regularity condition needed for Fenchel duality is satisfied for this problem due to the continuity of the norm function), we get

$$
\inf _{y \in X}\{\|y-x\|+\lambda g(y)\}=\max _{y^{*} \in X^{*}}\left\{-\|\cdot-x\|^{*}\left(-y^{*}\right)-(\lambda g)^{*}\left(y^{*}\right)\right\} .
$$

It is well known in convex analysis that

$$
\|\cdot-x\|^{*}\left(-y^{*}\right)= \begin{cases}\left\langle-y^{*}, x\right\rangle & \text { if }\left\|y^{*}\right\| \leq 1 \\ \infty & \text { otherwise }\end{cases}
$$

Implementing this into formula (4.9) and employing elementary transformations, we obtain

$$
\begin{aligned}
\inf _{y \in X}\{\|y-x\|+\lambda g(y)\} & =\max _{\left\|y^{*}\right\| \leq 1}\left\{\left\langle y^{*}, x\right\rangle-(\lambda g)^{*}\left(y^{*}\right)\right\} \\
& =\left\|y^{*}\right\| \leq 1,(\lambda g)^{*}\left(y^{*}\right) \leq \eta \\
& \left.\left.=\| y^{*}, x\right\rangle-\eta\right\} \\
& =\max _{\left\|y^{*}\right\| \leq 1, \lambda g^{*}\left(y^{*} / \lambda\right) \leq \eta}\left\{\left\langle y^{*}, x\right\rangle-\eta\right\} \\
& \max _{\left\|y^{*}\right\| \leq 1,(1 / \lambda)\left(y^{*}, \eta\right) \in \operatorname{epi} g^{*}}\left\{\left\langle y^{*}, x\right\rangle-\eta\right\} .
\end{aligned}
$$


The latter representation yields, by denoting $x^{*}:=(1 / \lambda) y^{*}$ and $\alpha:=(1 / \lambda) \eta$, that

$$
\inf _{y \in X}\{\|y-x\|+\lambda g(y)\}=\max _{\left(x^{*}, \alpha\right) \in \operatorname{epi} g^{*},\left\|x^{*}\right\| \leq 1 / \lambda} \lambda\left\{\left\langle x^{*}, x\right\rangle-\alpha\right\}
$$

Combining this with the formulas above, we arrive at

$$
\begin{aligned}
\operatorname{dist}(x ; Q) & =\max \left\{\max _{\left(x^{*}, \alpha\right) \in \text { epi } g^{*},\left\|x^{*}\right\| \leq 1 / \lambda} \lambda\left\{\left\langle x^{*}, x\right\rangle-\alpha\right\}, 0\right\} \\
& =\max _{\left(x^{*}, \alpha\right) \in \operatorname{epi} g^{*},\left\|x^{*}\right\| \leq 1 / \lambda}\left\{\lambda\left[\left\langle x^{*}, x\right\rangle-\alpha\right]_{+}\right\} .
\end{aligned}
$$

It is easy to observe the following relationships held for any $\lambda>0$ :

$\max _{(0, \alpha) \in \text { epi g* }} \lambda\{\langle 0, x\rangle-\alpha\}=\max _{g^{*}(0) \leq \alpha} \lambda(\langle 0, x\rangle-\alpha)=\lambda\left(-g^{*}(0)\right) \leq \lambda \inf _{x \in X} g(x) \leq \lambda g(\widehat{x})<0$.

Taking this into account, we have from (4.10) that

$$
\begin{aligned}
\operatorname{dist}(x ; Q) & =\max _{\left(x^{*}, \alpha\right) \in \text { epi } g^{*},\left\|x^{*}\right\| \leq 1 / \lambda}\left\{\lambda\left[\left\langle x^{*}, x\right\rangle-\alpha\right]_{+}\right\} \\
& =\max _{\left(x^{*}, \alpha\right) \in \operatorname{epi~} g^{*}} \max _{\left\|x^{*}\right\| \leq 1 / \lambda}\left\{\lambda\left[\left\langle x^{*}, x\right\rangle-\alpha\right]_{+}\right\} \\
& =\max _{\left(x^{*}, \alpha\right) \in \text { epi } g^{*}} \frac{\left[\left\langle x^{*}, x\right\rangle-\alpha\right]_{+}}{\left\|x^{*}\right\|},
\end{aligned}
$$

which gives (4.7) and thus completes the proof of the proposition.

Now we are ready we establish the required extension of the Ascoli formula (4.5) to the case of the infinite inequality systems under consideration.

Lemma 4.3 (distance to infinite linear inequalities in Banach spaces). Assume that the infinite system $\mathcal{F}(p)$ in (1.1) satisfies the strong Slater condition at $p=\left(p_{t}\right)_{t \in T}$. Then for any $x \in X$ and any $p \in l_{\infty}(T)$ we have the extended Ascoli distance formula

$$
\operatorname{dist}(x ; \mathcal{F}(p))=\max _{\left(x^{*}, \alpha\right) \in \mathrm{cl} \mathbf{l}^{*} C(p)} \frac{\left[\left\langle x^{*}, x\right\rangle-\alpha\right]_{+}}{\left\|x^{*}\right\|} .
$$

If furthermore $X$ is reflexive, then the distance formula (4.11) can be simplified as follows:

$$
\operatorname{dist}(x ; \mathcal{F}(p))=\sup _{\left(x^{*}, \alpha\right) \in C(p)} \frac{\left[\left\langle x^{*}, x\right\rangle-\alpha\right]_{+}}{\left\|x^{*}\right\|} .
$$

Proof. It is obvious to observe that the infinite system (1.1) is represented in

$$
\mathcal{F}(p=\{x \in X \mid g(x) \leq 0\}
$$

where the convex function $g: X \rightarrow \overline{\mathbb{R}}$ is given in the supremum form

$$
g(x):=\sup _{t \in T}\left(f_{t}(x)-p_{t}\right) \text { with } f_{t}(x):=\left\langle a_{t}^{*}, x\right\rangle-b_{t}
$$


The assumed strong Slater condition for $\mathcal{F}(p)$ ensures the fulfillment of the classical Slater condition for $g$ imposed in Proposition 4.2. To imply this proposition in the framework of (4.13), we need to compute the conjugate function to the supremum function in (4.14). The recent results in this direction $[4,18]$ yield that

$$
\text { epi } g^{*}=\operatorname{epi}\left\{\sup _{t \in T}\left(f_{t}-p_{t}\right)\right\}^{*}=\operatorname{cl} l^{*} \operatorname{co}\left(\bigcup_{t \in T} \operatorname{epi}\left(f_{t}-p_{t}\right)^{*}\right)=\operatorname{cl}^{*} C(p)+\mathbb{R}_{+}(0,1)
$$

with $0 \in X^{*}$, where the weak* closedness of the set $\mathrm{cl}^{*} C(p)+\mathbb{R}_{+}(0,1)$ is a consequence of the classical Dieudonné theorem; see, e.g., [39, Theorem 1.1.8]. Thus we get the distance formula (4.11) from Proposition 4.2 in general Banach spaces.

To prove the simplified distance formula (4.12) in the case of reflexive spaces, suppose on the contrary that it does not hold. Then there is a scalar $\beta \in \mathbb{R}$ such that

$$
\max _{\left(x^{*}, \alpha\right) \in \mathrm{c}^{*} C(p)} \frac{\left[\left\langle x^{*}, x\right\rangle-\alpha\right]_{+}}{\left\|x^{*}\right\|}>\beta>\sup _{\left(x^{*}, \alpha\right) \in C(p)} \frac{\left[\left\langle x^{*}, x\right\rangle-\alpha\right]_{+}}{\left\|x^{*}\right\|} .
$$

This yields the existence of a pair $\left(\bar{x}^{*}, \bar{\alpha}\right) \in \operatorname{cl}^{*} C(p)$ with $\bar{x}^{*} \in X^{*} \backslash\{0\}$ and $\bar{\alpha} \in \mathbb{R}$ such that

$$
\frac{\left[\left\langle\bar{x}^{*}, x\right\rangle-\bar{\alpha}\right]_{+}}{\left\|\bar{x}^{*}\right\|}>\beta \text {. }
$$

Taking into account that the space $X$ is reflexive and that the set $C(p)$ is convex and employing the Mazur weak closure theorem, we can replace the weak* closure of the $C(p)$ above by its norm closure in $X^{*}$. This allows us to find a sequence $\left(x_{k}^{*}, \alpha_{k}\right) \in C(p), k \in \mathbb{N}$, converging in norm to $\left(\bar{x}^{*}, \bar{\alpha}\right)$ as $k \rightarrow \infty$. Thus we get

$$
\lim _{k \rightarrow \infty} \frac{\left[\left\langle x_{k}^{*}, x\right\rangle-\alpha_{k}\right]_{+}}{\left\|x_{k}^{*}\right\|}=\frac{\left[\left\langle\bar{x}^{*}, x\right\rangle-\bar{\alpha}\right]_{+}}{\left\|\bar{x}^{*}\right\|}>\beta,
$$

and therefore there is $k_{0} \in \mathbb{N}$ such that

$$
\frac{\left[\left\langle x_{k_{0}}^{*}, x\right\rangle-\alpha_{k_{0}}\right]_{+}}{\left\|x_{k_{0}}^{*}\right\|}>\beta
$$

The latter surely contradicts (4.15) and this completes the proof of the lemma.

The following example shows that the reflexivity of the decision space $X$ is an essential requirement for the validity of the simplified distance formula (4.12), even in the framework of (nonreflexive) Asplund spaces.

Example 4.4 (failure of simplified distance formula in nonreflexive Asplund spaces). Consider the classical space $c_{0}$ of sequences of real numbers converging to zero endowed with the supremum norm. This space is well known to be Asplund while not reflexive; see, e.g., [17]. Let us show that the simplified distance formula (4.12) fails in $X=c_{0}$ for a rather plain linear system of countable inequalities. Of course, we need to demonstrate that the inequality " $\leq$ " is generally violated in (4.12), since the opposite inequality holds in any Banach space. Form the infinite (countable) linear inequality system

$$
\mathcal{F}(0):=\left\{x \in c_{0} \mid\left\langle e_{1}^{*}+e_{t}^{*}, x\right\rangle \leq-1, t \in \mathbb{N}\right\},
$$


where $e_{t}^{*} \in l_{1}$ has 1 as its $t$-th component while all the remaining components are 0 . System (4.16) can be rewritten as

$$
x \in \mathcal{F}(0) \Longleftrightarrow x(1)+x(t) \leq-1 \text { for all } t \in \mathbb{N} .
$$

Observe that for the origin $z=0$ we have $\operatorname{dist}(0 ; \mathcal{F}(0))=1$, and the distance is realized at, e.g., $u=(-1,0,0, \ldots)$. Indeed, passing to the limit in the inequality

$$
x(1)+x(t) \leq-1 \text { as } t \rightarrow \infty
$$

and taking into account that $x(t) \rightarrow 0$ as $t \rightarrow \infty$, by the structure of the space of $c_{0}$, we get $x(1) \leq-1$. Furthermore, it can be checked that

$$
\begin{aligned}
& \left(e_{1}^{*},-1\right) \in \mathrm{cl}^{*} C(0), \quad\left\langle e_{1}^{*}, x-u\right\rangle \leq 0 \text { for all } x \in \mathcal{F}(0), \\
& \operatorname{dist}(z ; \mathcal{F}(0))=\|z-u\|=\left\langle e_{1}^{*}, z-u\right\rangle=\frac{\left\langle e_{1}^{*}, z\right\rangle-(-1)}{\left\|e_{1}^{*}\right\|} .
\end{aligned}
$$

On the other hand, for the pair $\left(x^{*}, \alpha\right) \in X^{*} \times \mathbb{R}$ given by

$$
\left(x^{*}, \alpha\right):=\left(e_{1}^{*}+\sum_{t \in \mathbb{N}} \lambda_{t} e_{t}^{*},-1\right) \in C(0) \text { with } \lambda \in \mathbb{R}_{+}^{(\mathbb{N})} \text { and } \sum_{t \in \mathbb{N}} \lambda_{t}=1
$$

we can directly check that $\left\|x^{*}\right\|=2$ and hence

$$
\frac{\left[\left\langle x^{*}, z\right\rangle-\alpha\right]_{+}}{\left\|x^{*}\right\|}=\frac{1}{2}
$$

which shows that the equality in (4.12) is violated for the countable system (4.16) in the nonreflexive Asplund space $X=c_{0}$ of decision variables.

Our next step in to derive a verifiable precise formula for computing the exact Lipschitzian bound lip $\mathcal{F}(0, \bar{x})$ for the infinite system (1.1) in the general Banach space $X$. As a preliminary result we need the following technical lemma.

Lemma 4.5 (closed-graph property of the characteristic set mapping). The setvalued mapping $l_{\infty}(T) \ni p \mapsto \mathrm{cl}^{*} C(p) \subset X^{*} \times \mathbb{R}$ is closed-graph in the norm $\times$ weak ${ }^{*}$ topology of $\ell_{\infty}(T) \times\left(X^{*} \times \mathbb{R}\right)$, i.e., for any nets

$$
\left\{p_{\nu}\right\}_{\nu \in \mathcal{N}} \subset l_{\infty}(T), \quad\left\{x_{\nu}^{*}\right\}_{\nu \in \mathcal{N}} \subset X^{*}, \quad\left\{\beta_{\nu}\right\}_{\nu \in \mathcal{N}} \subset \mathbb{R}
$$

satisfying $p_{\nu} \rightarrow p, x_{\nu}^{*} \stackrel{w^{*}}{\longrightarrow} x^{*}, \beta_{\nu} \rightarrow \beta$, and $\left(x_{\nu}^{*}, \beta_{\nu}\right) \in \operatorname{cl}^{*} C\left(p_{\nu}\right)$ for every $\nu \in \mathcal{N}$ we have the inclusion $\left(x^{*}, \beta\right) \in \mathrm{cl}^{*} C(p)$.

Proof. Arguing by contradiction, suppose that $\left(x^{*}, \beta\right) \notin \mathrm{cl}^{*} C(p)$. Then the classical strict separation theorem allows us to find a pair $(x, \alpha) \in X \times \mathbb{R}$ with $(x, \alpha) \neq(0,0)$ and real numbers $\gamma$ and $\gamma^{\prime}$ satisfying

$$
\left\langle x^{*}, x\right\rangle+\beta \alpha<\gamma^{\prime}<\gamma \leq\left\langle a_{t}^{*}, x\right\rangle+\left(b_{t}+p_{t}\right) \alpha \text { for all } t \in T \text {. }
$$


Hence there exists a net index $\nu_{0} \in \mathcal{N}$ such that

$$
\left\langle x_{\nu}^{*}, x\right\rangle+\beta_{\nu} \alpha<\gamma^{\prime} \text { and }\left\|\alpha\left(p-p_{\nu}\right)\right\| \leq \gamma-\gamma^{\prime} \text { whenever } \nu \succeq \nu_{0}
$$

We have therefore that

$$
\begin{aligned}
\left\langle a_{t}^{*}, x\right\rangle+\alpha\left(b_{t}+p_{t \nu}\right) & =\left\langle a_{t}^{*}, x\right\rangle+\alpha\left(b_{t}+p_{t}\right)+\alpha\left(p_{t \nu}-p_{t}\right) \\
& \geq \gamma-\left\|\alpha\left(p_{\nu}-p\right)\right\| \geq \gamma^{\prime} \text { for all } t \in T .
\end{aligned}
$$

The latter implies that $\gamma^{\prime} \leq\left\langle z^{*}, x\right\rangle+\eta \alpha$ for all $\left(z^{*}, \eta\right) \in \mathrm{cl}^{*} C\left(p_{\nu}\right)$ whenever $\nu \succeq \nu_{0}$. Thus we arrive at the contradiction

$$
\left\langle x_{\nu}^{*}, x\right\rangle+\beta_{\nu} \alpha<\gamma^{\prime} \leq\left\langle x_{\nu}^{*}, x\right\rangle+\beta_{\nu} \alpha, \quad \nu \succeq \nu_{0},
$$

which completes the proof of the lemma.

Now we are ready to establish the desired formula for computing the exact Lipschitzian bound of $\mathcal{F}$ around $(0, \bar{x})$.

Theorem 4.6 (computing the exact Lipschitzian-bound). Let $\bar{x} \in \mathcal{F}(0)$ for the linear infinite inequality system (1.1) with a Banach decision space $X$. Assume that $\mathcal{F}$ satisfies the strong Slater condition at $p=0$ and that the coefficient set $\left\{a_{t}^{*} \mid t \in T\right\}$ is bounded in $X^{*}$. The following assertions hold:

(i) If $\bar{x}$ is a strong Slater point for $\mathcal{F}$ at $p=0$, then $\operatorname{lip} \mathcal{F}(0, \bar{x})=0$.

(ii) If $\bar{x}$ is not a strong Slater point for $\mathcal{F}$ at $p=0$, then the exact Lipschitzian bound of $\mathcal{F}$ around $(0, \bar{x})$ is computed by

$$
\operatorname{lip} \mathcal{F}(0, \bar{x})=\max \left\{\left\|x^{*}\right\|^{-1} \mid\left(x^{*},\left\langle x^{*}, \bar{x}\right\rangle\right) \in \operatorname{cl}^{*} C(0)\right\}>0
$$

via the $w^{*}$-closure of the characteristic set (2.8) at $p=0$.

Proof. Let us first justify (i). We have seen in the proof of Theorem 3.5(i) that our current assumptions imply that $(0, \tilde{x}) \in \operatorname{int}(\operatorname{gph} \mathcal{F})$, which in turn yields by the definition of the exact Lipschitzian bound that $\operatorname{lip} \mathcal{F}(0, \bar{x})=0$ in this case.

Next we prove the more difficult assertion (ii) of the theorem assuming that $\bar{x}$ is not a strong Slater point for $\mathcal{F}$ at $p=0$. Observe that by Lemma 3.4 the set (3.8) under the maximum operation on the right-hand side in (4.17) is nonempty and $w^{*}$-compact in $X^{*}$ and the maximum over this set is realized and hence it is finite. The inequality " $\geq$ " in (4.17) follows from the estimate

$$
\operatorname{lip} \mathcal{F}(0, \bar{x}) \geq\left\|D^{*} \mathcal{F}(0, \bar{x})\right\|
$$

established for general mappings between Banach space in [31, Theorem 1.44] and from formula (3.9) for computing the coderivative norm of the inequality system $\mathcal{F}$ in (1.1) derived above in Theorem 3.5. It remains to prove the opposite inequality " $\leq$ " in (4.17).

To proceed, let $M:=\sup _{t \in T}\left\|a_{t}^{*}\right\|<\infty$ and observe that the inequality " $\leq$ " in (4.17) is obvious when $L:=\operatorname{lip} \mathcal{F}(0, \bar{x})=0$. Suppose now that $L>0$ and consider any pair $(p, x)$ 
sufficiently close to $(0, \bar{x})$ in the limiting representation (2.2) of the exact Lipschitzian bound $\operatorname{lip} \mathcal{F}(0, \bar{x})$. We can confine ourselves to the case of $(p, x) \notin \operatorname{gph} \mathcal{F}$ by $L>0$. Furthermore, it follows from the structure of $\mathcal{F}$ in (1.1) that

$$
0<\operatorname{dist}\left(p ; \mathcal{F}^{-1}(x)\right)=\sup _{t \in T}\left[\left\langle a_{t}^{*}, x\right\rangle-b_{t}-p_{t}\right]_{+} .
$$

Moreover we have the relationships

$$
\begin{aligned}
\left\langle a_{t}^{*}, x\right\rangle-b_{t}-p_{t} & =\left\langle a_{t}^{*}, x-\bar{x}\right\rangle+\left\langle a_{t}^{*}, \bar{x}\right\rangle-b_{t}-p_{t} \\
& \leq M\|x-\bar{x}\|+\|p\| \text { for all } t \in T
\end{aligned}
$$

which allow us to conclude that

$$
\begin{aligned}
0 & <\sup _{\left(x^{*}, \beta\right) \in \mathrm{cl} \mathrm{l}^{*} C(p)}\left[\left\langle x^{*}, x\right\rangle-\beta\right]_{+}=\sup _{\left(x^{*}, \beta\right) \in \mathrm{c} \mathrm{l}^{*} C(p)}\left\{\left\langle x^{*}, x\right\rangle-\beta\right\} \\
& \leq M\|x-\bar{x}\|+\|p\| .
\end{aligned}
$$

Consider further the set

$$
C_{+}(p, x):=\left\{\left(x^{*}, \beta\right) \in \mathrm{cl}^{*} C(p) \mid\left\langle x^{*}, x\right\rangle-\beta>0\right\}
$$

which is obviously nonempty, and denote

$$
M_{(p, x)}:=\sup \left\{\left\|x^{*}\right\|^{-1} \mid\left(x^{*}, \beta\right) \in C_{+}(p, x)\right\} .
$$

In our setting we have $0 \in \operatorname{int}(\operatorname{dom} \mathcal{F})$ (cf. Lemma 2.3 and Remark 2.4), and therefore $p \in$ $\operatorname{dom} \mathcal{F}$ for all $p \in \ell_{\infty}(T)$ sufficiently close to the origin. In this case the set $C_{+}(p, x)$ cannot contain any element of the form $(0, \beta)$, since the contrary would yield by the definition of $C_{+}(p, x)$ that $\beta<0$, while Lemma 2.1 gives $\beta \geq 0$. Thus we get $0<\left\|x^{*}\right\| \leq M$ whenever $\left(x^{*}, \beta\right) \in C_{+}(p, x)$ and, in particular, $M_{(p, x)} \in(0, \infty]$. It follows furthermore that

$$
\frac{\sup _{\left(x^{*}, \beta\right) \in \mathrm{cl}^{*} C(p)} \frac{\left[\left\langle x^{*}, x\right\rangle-\beta\right]_{+}}{\left\|x^{*}\right\|}}{\sup _{\left(x^{*}, \beta\right) \in \mathrm{cl}^{*} C(p)}\left[\left\langle x^{*}, x\right\rangle-\beta\right]_{+}}=\frac{\sup _{\left(x^{*}, \beta\right) \in \mathrm{cl}^{*} C(p)} \frac{\left\langle x^{*}, x\right\rangle-\beta}{\left\|x^{*}\right\|}}{\sup _{\left(x^{*}, \beta\right) \in \mathrm{cl} \mathrm{l}^{*} C(p)}\left\{\left\langle x^{*}, x\right\rangle-\beta\right\}} \leq M_{(p, x)},
$$

where the latter inequality implies the estimate

$$
L \leq \limsup _{(p, x) \rightarrow(0, \bar{x}), x \notin \mathcal{F}(p) \neq \emptyset} M_{(p, x)}:=K
$$

Considering next a sequence $\left(p_{k}, x_{k}\right) \rightarrow(0, \bar{x})$ with $x_{k} \notin \mathcal{F}\left(p_{k}\right) \neq \emptyset$ and

$$
L \leq \lim _{k \rightarrow \infty} M_{\left(p_{k}, x_{k}\right)}=K,
$$

we select a sequence $\left\{\alpha_{k}\right\}_{k=1}^{\infty} \subset \mathbb{R}$ such that

$$
\lim _{k \rightarrow \infty} \alpha_{k}=K \text { and } 0<\alpha_{k}<M_{\left(p_{k}, x_{k}\right)} \text { as } k \in \mathbb{N} \text {. }
$$


Take now $\left(x_{k}^{*}, \beta_{k}\right) \in C_{+}\left(p_{k}, x_{k}\right)$ with $\alpha_{k}<\left\|x_{k}^{*}\right\|^{-1}$ for all $k \in \mathbb{N}$. Since the sequence $\left\{x_{k}^{*}\right\}_{k \in \mathbb{N}} \subset X^{*}$ is bounded, it contains a subnet $\left\{x_{\nu}^{*}\right\}_{\nu \in \mathcal{N}}$ that $w^{*}$-converges to some $x^{*} \in$ $X^{*}$. Denoting by $\left\{p_{\nu}\right\},\left\{x_{\nu}\right\},\left\{\beta_{\nu}\right\}$, and $\left\{\alpha_{\nu}\right\}$ the corresponding subnets of $\left\{p_{k}\right\},\left\{x_{k}\right\}$, $\left\{\beta_{k}\right\}$, and $\left\{\alpha_{k}\right\}$, we get from (4.19) that

$$
0<\left\langle x_{\nu}^{*}, x_{\nu}\right\rangle-\beta_{\nu} \leq M\left\|x_{\nu}-\bar{x}\right\|+\left\|p_{\nu}\right\|
$$

Thus $\left\langle x_{\nu}^{*}, x_{\nu}\right\rangle-\beta_{\nu} \rightarrow 0$, which implies by the construction above that $\beta_{\nu} \rightarrow\left\langle x^{*}, \bar{x}\right\rangle$. By Lemma 4.5 we get that

$$
\left(x^{*},\left\langle x^{*}, \bar{x}\right\rangle\right) \in \mathrm{cl}^{*} C(0),
$$

and then Lemma 2.3 ensures that $x^{*} \neq 0$.

To conclude proving the inequality " $\leq$ " in (4.17), observe that

$$
\left\|x^{*}\right\| \leq \liminf _{\nu \in \mathcal{N}}\left\|x_{\nu}^{*}\right\| \leq \lim _{\nu} \frac{1}{\alpha_{\nu}}=\frac{1}{K}
$$

due to $\left\|x_{\nu}^{*}\right\| \leq \alpha_{\nu}^{-1}$ and $\lim _{\nu} \alpha_{\nu}=K$, which gives

$$
L \leq K \leq \frac{1}{\left\|x^{*}\right\|} \leq \max \left\{\left\|z^{*}\right\|^{-1} \mid\left(z^{*},\left\langle z^{*}, \bar{x}\right\rangle\right) \in \mathrm{cl}^{*} C(0)\right\} .
$$

Remembering the notation above, we complete the proof of the theorem.

Comparing finally the results on computing the coderivative norm in Theorem 3.5 and the exact Lipschitzian bound in Theorem 4.6 allows us to arrive at the unconditional relationship between the coderivative norm and exact Lipschitzian bound of the infinite inequality system $\mathcal{F}$ with arbitrary Banach decision spaces. This was known before only for set-valued mappings between finite-dimensional spaces; $c f$. formula (2.5) and the corresponding discussions is Section 2.

Corollary 4.7 (relationship between the exact Lipschitzian bound and coderivative norm). Let $\breve{x} \in \mathcal{F}(0)$ for the infinite system (1.1) satisfying the strong Slater condition at $p=0$. Assume that the decision space $X$ is arbitrary Banach and that the coefficient set $\left\{a_{t}^{*} \mid t \in T\right\}$ is bounded in $X^{*}$. Then

$$
\operatorname{lip} \mathcal{F}(0, \bar{x})=\left\|D^{*} \mathcal{F}(0, \bar{x})\right\|
$$

Proof. If $\vec{x}$ is a strong Slater point for $\mathcal{F}$ at $p=0$, then we get equality (4.20) directly by comparing assertions (i) in Theorem 3.5 and Theorem 4.6 that ensure that

$$
\operatorname{lip} \mathcal{F}(0, \bar{x})=\left\|D^{*} \mathcal{F}(0, \bar{x})\right\|=0 .
$$

On the other hand, if $\bar{x}$ is not a strong Slater point for $\mathcal{F}$ at $p=0$, then (4.20) follows from comparing assertions (ii) in Theorem 3.5 and Theorem 4.6 that justify the same formula for computing $\left\|D^{*} \mathcal{F}(0, \bar{x})\right\|$ and $\operatorname{lip} \mathcal{F}(0, \bar{x})$ in (3.9) and (4.17), respectively.

Acknowledgement. The main results of the paper were obtained in our Research Report [10], which was widely dissimilated in the optimization community. We would like 
to thank many colleagues and friends for their helpful comments and remarks. Especially important contributions came from Radu Bot,, who suggested a new proof of Lemma 4.3 via Proposition 4.2 allowing us to remove a certain boundedness assumption imposed in [10], and from Constantin Zălinescu whose various deep insights led us, in particular, to simplified and more elegant proofs of Theorems 3.5 and 4.6 in comparison with the original ones given in [10]. We also gratefully indebted to two anonymous referees and the handling Associate Editor Jiři Outrata for their valuable suggestions and remarks that allowed us to essentially improve the original presentation.

\section{References}

[1] E. J. Anderson And P. NAsh, Linear Programming in Infinite-Dimensional Spaces, Wiley, Chicherster, 1987.

[2] G. BeER, Topologies on Closed and Closed Convex Sets, Kluwer, Dordrecht, 1993.

[3] J. M. Borwein AND Q. J. ZHU, Techniques of Variational Analysis, Springer, New York, 2005 .

[4] R. I. Bot?, S. M. GRAD AND G. WANKA, Generalized Moreau-Rockafellar results for composed convex functions, Optimization, to appear.

[5] B. Brosowski, Parametric Semi-Infinite Optimization, Peter Lang, Frankfurt, 1982.

[6] M. J. Cánovas, A. L. Dontchev, M. A. López and J. Parra, Metric regularity of semi-infinite constraint systems, Math. Program., 104 (2005), pp. 329-346.

[7] M. J. Cánovas, F. J. Gómez-Senent and J. Parra, Regularity modulus of arbitrarily perturbed linear inequality systems, J. Math. Anal. Appl., 343 (2008), pp. 315-327.

[8] M. J. Cánovas, A. Hantoute, M. A. López and J. Parra, Lipschitz modulus in convex semi-infinite optimization via DC functions, ESAIM: Control Optim. Calc. Var., published online (2008).

[9] M. J. Cánovas, D. Klatte, M. A. López AND J. Parra, Metric regularity in convex semi infinite optimization under canonical perturbations, SIAM J. Optim., 18 (2007), pp. 717-732.

[10] M. J. Cánovas, M. A. López, B. S. Mordukhovich and J. Parra, Robust stability and optimality conditions for parametric infiniyte and semi-infinite programs, Research Report \# 12 (2008), 32 pp., Department of Mathematics, Wayne State University, Detroit, MI.

[11] M. J. CÁnovas, M. A. López, B. S. Mordukhovich and J. Parra, Variational analysis in semi-infinite and infinite programming, II: Necessary optimality conditions, submitted (2009). 
[12] M. J. Cánovas, M. A. Lopez, J. Parra and M. I. Todorov, Stability and wellposedness in linear semi-infinite programming, SIAM J. Optim., 10 (1999), pp. 82-99.

[13] N. Dinh, M. A. Goberna And M. A. López, From linear to convex systems: Consistency, Farkas' lemma and applications, J. Convex Anal., 13 (2006), pp. 279-290.

[14] N. Dinh, M. A. Goberna And M. A. López, On the stability of the feasible set in optimization problems, submitted (2008).

[15] N. Dinh, B. S. Mordukhovich And T. T. A. NGHIA, Subdifferentials of value functions and optimality conditions for $D C$ and bilevel infinite and semi-infinite programs, Math. Program., to appear.

[16] N. Dunford and J. T. SchwarTz, Linear Operators Part I: General Theory, Wiley, New York, 1988.

[17] M. Fabian, P. Habala, P. Hájek, V. Montesinos-Santalucía, J. Pelant and V. ZIZLER, Functional Analysis and Infinite-Dimensional Geometry, Springer, New York, 2001.

[18] M. A. Goberna, V. Jeyakumar and M. A. LóPez, Necessary and sufficient constraint qualifications for solvability of systems of infinite convex inequalities, Nonlinear Anal., 68 (2008), pp. 1184-1194

[19] M. A. Goberna And M. A. López, Linear Semi-Infinite Optimization, Wiley, Chichester, 1998.

[20] A. D. IofFe, Metric regularity and subdifferential calculus, Russian Math. Surv., 55 (2000), pp. 501-558.

[21] H. T. Jongen, J.-J. RÜCKMANN AND G.-W. WEBER, One-parametric semi-infinite optimization: On the stability of the feasible set, SIAM J. Optim., 4 (1994), pp. 637648.

[22] D. Klatte and R. Henrion, Regularity and stability in nonlinear semi-infinite optimization, in Semi-Infinite Programming, R. Reemtsen and J.-J. Rückmann, eds., Kluwer, Dordrecht, 1998, pp. 69-102.

[23] D. Klatte and B. Kummer, Nonsmooth Equations in Optimization. Regularity, Calculus, Methods and Applications, Kluwer, Dordrecht, 2002.

[24] C. LI AND K. F. NG, On constraint qualification for an infinite system of convex inequalities in a Banach space, SIAM J. Optim., 15 (2005), pp. 488-512.

[25] C. LI, K. F. NG AND T. K. Pong, Constraint qualifications for convex inequality systems with applications to constrained optimization, SIAM J. Optim., 19 (2008), pp. 163-187.

[26] B. S. MoRdukHovich, Maximum principle in problems of time optimal control with nonsmooth constraints, J. Appl. Math. Mech., 40 (1976), pp. 960-969. 
[27] B. S. MoRDUKHOVICH, Metric approximations and necessary optimality conditions for general classes of extremal problems, Soviet Math. Dokl., 22 (1980), pp. 526-530.

[28] B. S. MoRdukHovich, Complete characterizations of openness, metric regularity, and Lipschitzian properties of multifunctions, Trans. Amer. Math. Soc., 340 (1993), pp. $1-35$.

[29] B. S. MoRdUKHovich, Coderivatives of set-valued mappings: Calculus and applications, Nonlinear Anal., 30 (1997), pp. 3059-3070.

[30] B. S. MoRdukHovich, Necessary conditions in nonsmooth minimization via lower and upper subgradients, Set-Valued Anal., 12 (2004), 163-193.

[31] B. S. MoRdukhovich, Variational Analysis and Generalized Differentiation, I: Basic Theory, Springer, Berlin, 2006.

[32] B. S. Mordukhovich, Variational Analysis and Generalized Differentiation, II: Applications, Springer, Berlin, 2006.

[33] J. V. OUTRATA, Mathematical programs with equilibrium-constraints:-Theory and numerical methods, in Nonsmooth Mechanics of Solids, J. Haslinger and G. E. Stavroulakis, eds., Springer, New York, 2006, pp. 221-274.

[34] R. R. Phelps, Convex Functions, Monotone Operators and Differentiability, 2nd edition, Springer, Berlin, 1993.

[35] R. T. Rockafellar and R. J-B. Wets, Variational Analysis, Springer, Berlin, 1998.

[36] W. SchirotZeK, Nonsmooth Analysis, Springer, Berlin, 2007.

[37] O. Stein, Bilevel Strategies in Semi-Infinite Programming, Kluwer, Boston, 2003.

[38] C. ZăLINESCU, Solvability results for sublinear functions and operators, Z. Oper. Res. Ser. A, 31 (1987), pp. 79-101.

[39] C. ZăLInescU, Convex Analysis in General Vector Spaces, World Scientific, Singapore, 2002. 